\title{
Resetting proteostasis with ISRIB prevents pulmonary fibrosis
}

Satoshi Watanabe ${ }^{1,2}$, Nikolay S. Markov ${ }^{1}$, Ziyan Lu ${ }^{1}$, Raul Piseaux Aillon ${ }^{1}$, Saul Soberanes ${ }^{1}$, Constance E.

Runyan $^{1}$, Ziyou Ren ${ }^{1}$, Rogan A. Grant ${ }^{1}$, Mariana Maciel $^{1}$, Hiam Abdala-Valencia ${ }^{1}$, Yuliya Politanska ${ }^{1}$, Kiwon Nam $^{1}$, Lango Sichizya ${ }^{1}$, Hermon G. Kihshen ${ }^{1}$, Nikita Joshi ${ }^{1}$, Alexandra C. McQuattie-Pimentel ${ }^{1}$, Richard I. Morimoto $^{3}$, Paul A. Reyfman 1 , G.R. Scott Budinger ${ }^{1,4}$, Alexander V. Misharin ${ }^{1,4}$

${ }^{1}$ Department of Medicine, Division of Pulmonary and Critical Care Medicine, Northwestern

University Feinberg School of Medicine, Chicago, IL, 60611, USA

${ }^{2}$ Department of Respiratory Medicine, Kanazawa University Graduate School of Medical Sciences, Kanazawa, 920-8641, Japan

${ }^{3}$ Deparment of Molecular Biosciences, Northwestern University, Evanston, IL 60208

${ }^{4}$ These senior authors contributed equally

Running title: ISRIB ameliorates pulmonary fibrosis in aging

Corresponding author:

G.R. Scott Budinger

Department of Medicine, Division of Pulmonary and Critical Care Medicine, Northwestern University, Feinberg School of Medicine

303 E Superior, Simpson Querrey Biomedical Research Center, 5th floor, Chicago, IL, 60611, USA

E-mail: s-buding@northwestern.edu

Phone: 312-908-7737, Fax: 312-503-0411

Alexander V. Misharin

Department of Medicine, Division of Pulmonary and Critical Care Medicine, Northwestern University, Feinberg School of Medicine

303 E Superior, Simpson Querrey Biomedical Research Center, 5th floor, Chicago, IL, 60611, USA

E-mail: a-misharin@northwestern.edu

Phone: 312-908-7737, Fax: 312-503-0411 


\begin{abstract}
Aging is among the most important risk factors for the development of pulmonary fibrosis. We found that a small molecule that specifically inhibits translational inhibition induced by activation of the integrated stress response (ISRIB) attenuated the severity of pulmonary fibrosis in young and old mice. The more severe fibrosis in old compared to young mice was associated with increased recruitment of pathogenic monocyte-derived alveolar macrophages. Using genetic lineage tracing and transcriptomic profiling we found that ISRIB modulates stress response signaling in alveolar epithelial cells resulting in decreased apoptosis and decreased recruitment of pathogenic monocyte-derived alveolar macrophages. These data support multicellular model of fibrosis involving epithelial cells, pathogenic monocyte-derived alveolar macrophages and fibroblasts.

Inhibition of the integrated stress response in the aging lung epithelium ameliorates pulmonary fibrosis by preventing the prolonged recruitment of monocyte-derived alveolar macrophages.
\end{abstract}

Keywords: pulmonary fibrosis, aging, integrated stress response, ISRIB 


\section{INTRODUCTION}

Pulmonary fibrosis is a relentlessly progressive and often fatal disease characterized by the replacement of normal lung tissue with fibrotic scar that increases the work of breathing and impairs gas exchange $(1,2)$. Advanced age is among the most important risk factors for the development of pulmonary fibrosis (3). Even in patients with a genetic predisposition, the onset of disease seldom occurs before the sixth decade and the incidence increases exponentially with advancing age $(4,5)$. The development of cellular senescence, mitochondrial dysfunction, stem cell exhaustion and proteostatic stress have all been implicated in the agerelated susceptibility to pulmonary fibrosis, but the molecular mechanisms linking these pathways with the development of fibrosis are poorly understood $(6,7)$.

Proteostasis refers to the dynamic process by which cells control the concentration, conformation, binding interactions, and stability of individual proteins making up the proteome via regulated networks that influence protein synthesis, folding, trafficking, disaggregation, and degradation $(8,9)$. Analyses of families with high rates of pulmonary fibrosis as well as unbiased genetic studies in populations of patients with idiopathic pulmonary fibrosis (IPF) identified associations between mutations in genes predicted to disrupt proteostasis in the alveolar epithelium and the development of pulmonary fibrosis (10). Proteostatic stress activates the intracellular sensors PERK, PKR, GCN2 or HRI to induce the integrated stress response (ISR) (11). Activation of the ISR reduces the rate of global protein translation while enhancing the translation of selected transcription factors, including ATF4 and CHOP, that induce the expression of chaperones and other cell-protective genes. Prolonged or high-level induction of CHOP activates apoptotic pathways that result in cell death (12). The ISR is also activated via PKR in response to viral infections, which have been implicated in exacerbations of IPF (13).

We and others have suggested that proteostatic stress induced by genetic mutations or environmental agents that drive fibrosis leads to the recruitment of profibrotic, monocyte-derived alveolar macrophages to the lung (1425). We have shown that these monocyte-derived alveolar macrophages are maintained by autocrine or paracrine signaling via M-CSF/M-CSFR and localized specifically to regions of epithelial injury in close proximity to fibroblasts, inducing their activation, proliferation and secretion of matrix proteins (18). Over time, these regulatory circuits between macrophages and fibroblasts may become dispensable, creating autonomous regions of progressive fibrosis driven exclusively by fibroblasts $(26,27)$. Even so, therapeutic strategies that reduce the ongoing recruitment of monocyte-derived alveolar macrophages and prevent the initiation of macrophage-fibroblast circuits are likely to be effective as the progression of the human disease is heterogenous in space and time. 
We found that enhanced and prolonged fibrosis in old compared with young mice was associated with prolonged recruitment of pathogenic monocyte-derived alveolar macrophages suggesting a failure to repair epithelial injury in older animals. A small molecule integrated stress response inhibitor (ISRIB) specifically prevents translational inhibition and therefore other downstream consequences of activation of the ISR and has shown promise as a therapeutic strategy against inflammation, cancer and neurocognitive diseases in aging (28, 29). We found that ISRIB attenuated the development of fibrosis in young and aged animals in resolving (bleomycin) and non-resolving (asbestos) models of pulmonary fibrosis. The administration of ISRIB ameliorated pulmonary fibrosis by increasing the resilience of epithelial cells to proteostatic stress, preventing their apoptosis and attenuating the recruitment of pathogenic monocyte-derived alveolar macrophages. 


\section{RESULTS}

\section{Aging is associated with more severe pulmonary fibrosis in response to bleomycin and asbestos.}

We induced pulmonary fibrosis in young mice (3-months) and old mice (over 18-months) via the intratracheal administration of bleomycin $(0.025 \mathrm{unit} / 50 \mu \mathrm{l})$, and analyzed the lungs 28 days later (Figure 1A-D). Old mice developed more severe pulmonary fibrosis compared with young mice, as evidenced by decreased lung compliance, elevated collagen levels, and worsened lung pathology (Figure 1A and B). While collagen levels in young mice returned to the near-normal levels, increased collagen levels persisted in old mice for up to 56 days (Figure 1C). Both young and old mice lost body weight after the instillation of bleomycin. However, while body weight recovered in young mice, it failed to recover in old animals (Figure 1D).

We observed a similar age-related susceptibility in a non-resolving model of pulmonary fibrosis induced by the intratracheal administration of crocidolite asbestos $(100 \mu \mathrm{g} / 50 \mu \mathrm{l})(18,30)$. In young mice exposed to intratracheal asbestos, we observed mild peribronchial fibrosis as evidenced by histological scoring of Masson's trichrome stained lung sections, but body weight and lung compliance were not changed (Figure 1E-H). In contrast, old animals lost and did not recover body weight and had reduced lung compliance, increased lung collagen levels and increased fibrosis area as evident by Masson's trichrome staining 28 days after asbestos administration (Figure 1E-H). Collagen levels remained increased up to 56 days after asbestos administration (Figure 1G). Thus, aging is associated with increased severity of fibrosis in both bleomycin- and asbestosinduced models of pulmonary fibrosis. 
bioRxiv preprint doi: https://doi.org/10.1101/2020.02.26.965566; this version posted February 27, 2020. The copyright holder for this preprint (which was not certified by peer review) is the author/funder. All rights reserved. No reuse allowed without permission.

A Young, Bleomycin

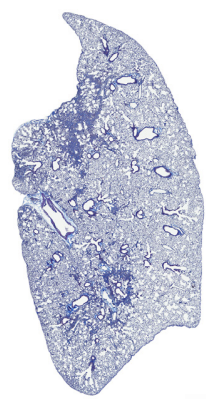

Old, Bleomycin

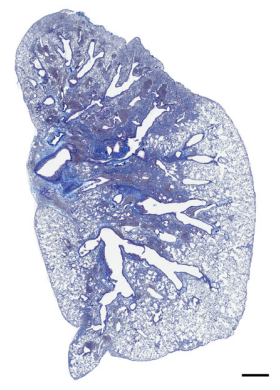

C

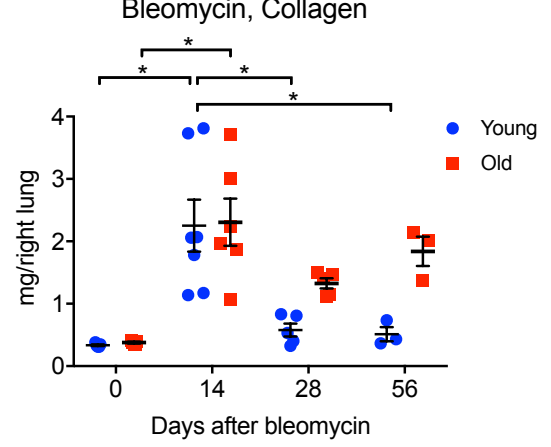

E

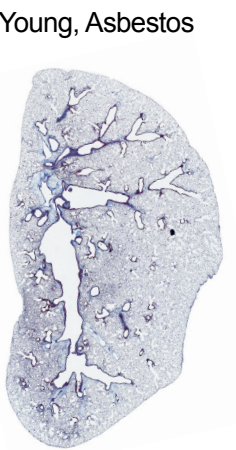

Old, Asbestos

G

Asbestos, Collagen

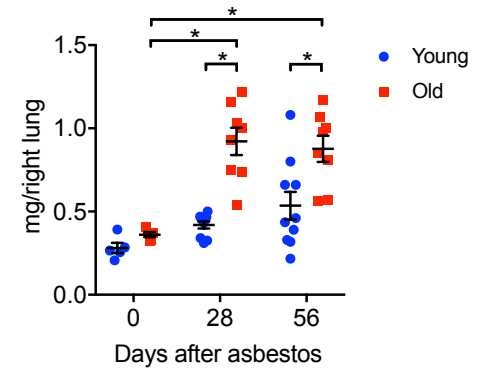

B

Fibrosis score
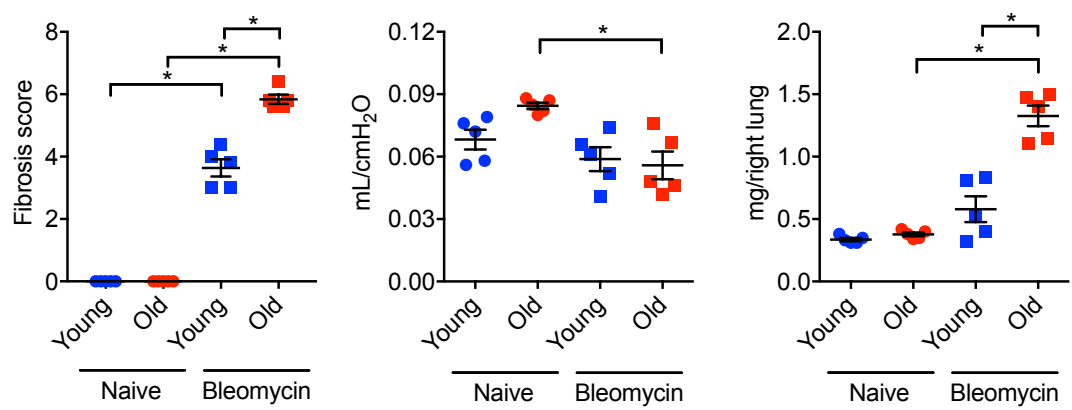

D

Young, Bleomycin
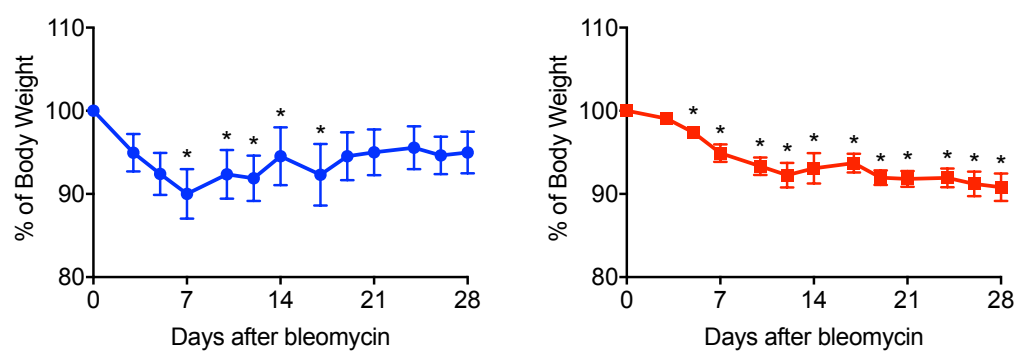

F

Fibrosis score

Lung compliance

Collagen
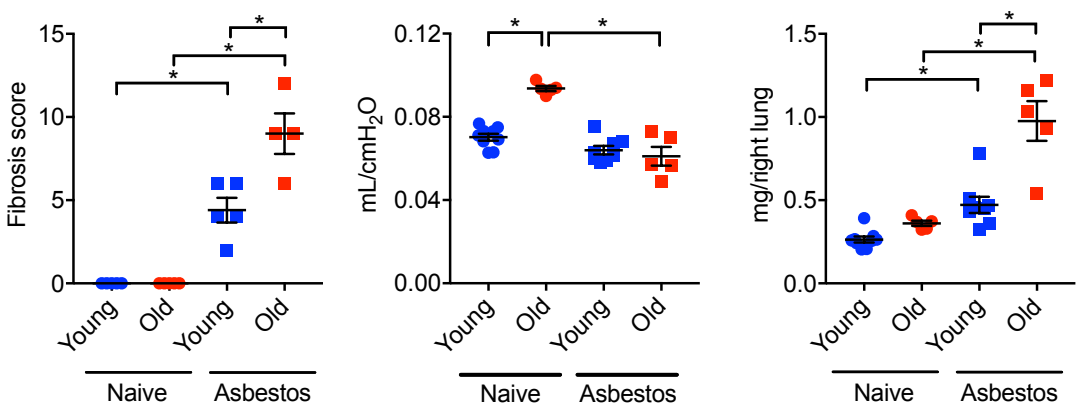

$\mathrm{H}$

Young, Asbestos

Old, Asbestos
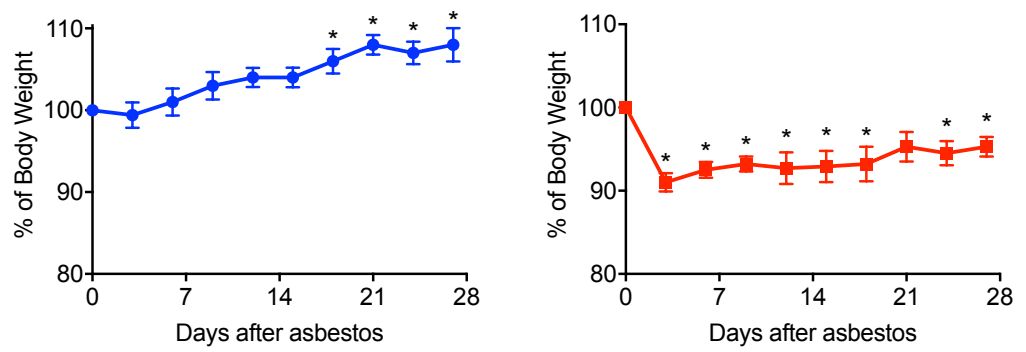
Figure 1. Old mice develop more severe lung fibrosis compared to young mice. Young mice ( 3 months) and old mice (over 18 months) were administered bleomycin $(0.025$ unit/50 $\mu \mathrm{l})$ or crocidolite asbestos $(100 \mu \mathrm{g} / 50$ $\mu 1$ ) intratracheally and the lungs were harvested 28 days later. (A) Representative histologic images in bleomycin model (Masson's trichrome, Scale bar = $1 \mathrm{~mm}$ ). (B) Quantification of fibrosis score using Ashcroft score, lung compliance using Flexivent, and soluble collagen in lung homogenates in bleomycin model. (C) Quantification of soluble collagen in naïve mice and 14, 28, and 56 days after the administration of bleomycin. (D) Body weight in bleomycin model with young and old mice. (E) Representative histologic images in asbestos model (Masson's trichrome, Scale bar $=1 \mathrm{~mm}$ ). $(\mathbf{F})$ Quantification of fibrosis score, lung compliance using Flexivent, and soluble collagen in lung homogenates in asbestos model. (G) Quantification of soluble collagen in naïve mice and 28 and 56 days after the administration of asbestos. (H) Body weight in asbestos model with young and old mice. Data presented as mean \pm SEM, 5-10 mice per group, one-way ANOVA with Tukey-Kramer test for multiple comparisons or Kruskal-Wallis test with Dunn's multiple comparisons test for comparison of each body weight to baseline (day 0 ). ${ }^{*}, \mathrm{p}<0.05$. Representative data from two independent experiments. 


\section{Recruitment of pathogenic monocyte-derived alveolar macrophages is increased during pulmonary fibrosis in old mice.}

We and others have performed causal genetic studies in mice to show that monocyte-derived alveolar macrophages are necessary for the development of bleomycin- and asbestos-induced pulmonary fibrosis independent of circulating monocytes, tissue-resident interstitial macrophages or tissue-resident alveolar macrophages (14-18). Therefore, we asked whether the increased severity of pulmonary fibrosis in old mice is associated with an increased recruitment of monocyte-derived alveolar macrophages. We quantified myeloid cell populations in the lung via flow cytometry 28 days after the administration of bleomycin or asbestos, as previously described (16) (Figure 2 and Figure S1). We used differential expression of Siglec F to separate monocyte-derived alveolar macrophages $\left(\mathrm{CD}^{+} 4^{+}\right.$Siglec $\left.\mathrm{F}^{\text {low }}\right)$ from tissue-resident alveolar macrophages $\left(\mathrm{CD}^{+} 4^{+}\right.$Siglec $\left.\mathrm{F}^{\text {high }}\right)(16,31)$. We found that numbers of classical monocytes and lymphocytes were increased in the lungs of old mice both in the steady state and during asbestos-induced fibrosis, as has been reported (Figure 2 and Figure S2) (32). After the intratracheal instillation of either bleomycin or asbestos, the number of interstitial macrophages $\left(\mathrm{CD}^{+} 4^{+}\right.$Siglec $\left.\mathrm{F}^{-}\right)$and monocyte-derived alveolar macrophages was increased in old compared to young mice (Figure 2). In our gating strategy interstitial macrophages include a mixed population of tissue-resident perivascular and peribronchial macrophages and differentiating monocyte-derived macrophages. Thus, the increased severity of pulmonary fibrosis in old mice was associated with increased numbers of profibrotic monocyte-derived alveolar macrophages. 
A Bleomycin
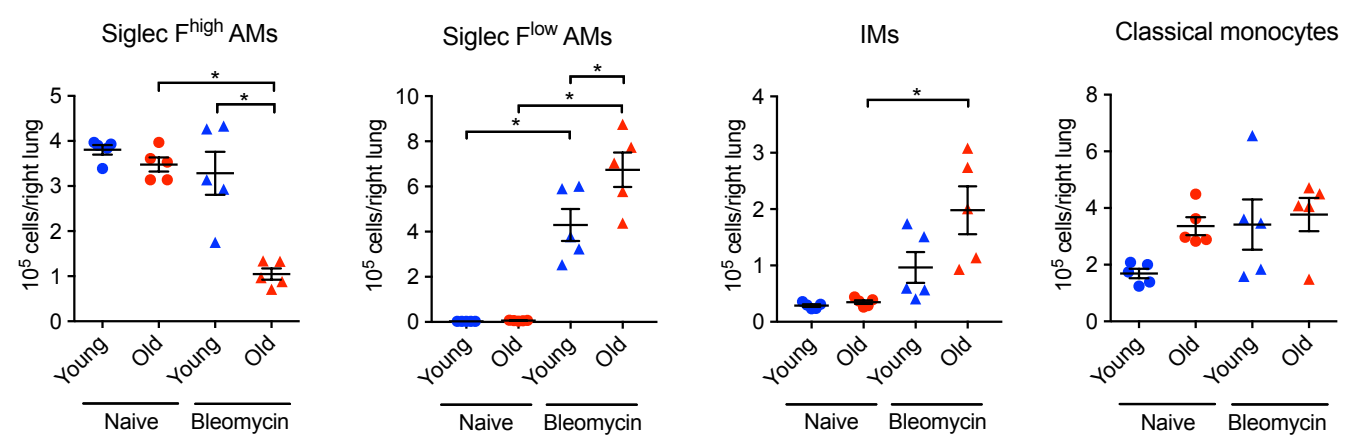

B Asbestos
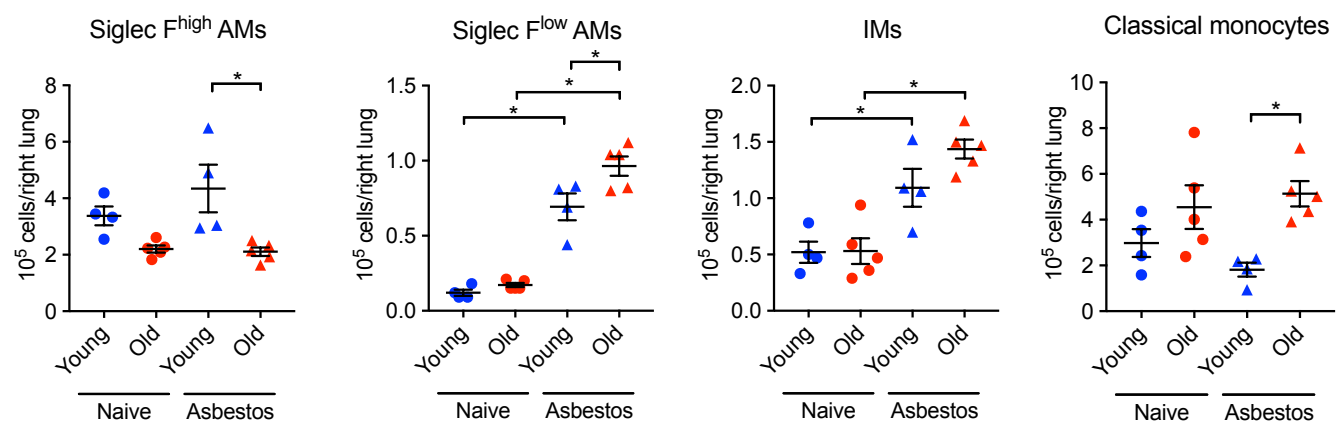

Figure 2. Aging is associated with the increased number of monocyte-derived alveolar macrophages in

lung fibrosis. Young mice (3 months) and old mice (over 18 months) were administered bleomycin $(0.025$ unit/50 $\mu$, intratracheally) or crocidolite asbestos (100 $\mu \mathrm{g} / 50 \mu \mathrm{l}$, intratracheally), and the lungs were harvested 21 days later. Flow cytometry to quantify monocyte and macrophage populations in (A) bleomycin model and (B) asbestos model. Data presented as mean \pm SEM, 5-8 mice per group, one-way ANOVA with TukeyKramer test for multiple comparisons. *, $p<0.05$. Representative data from two independent experiments. 
A Gating strategy (myeloid cells)
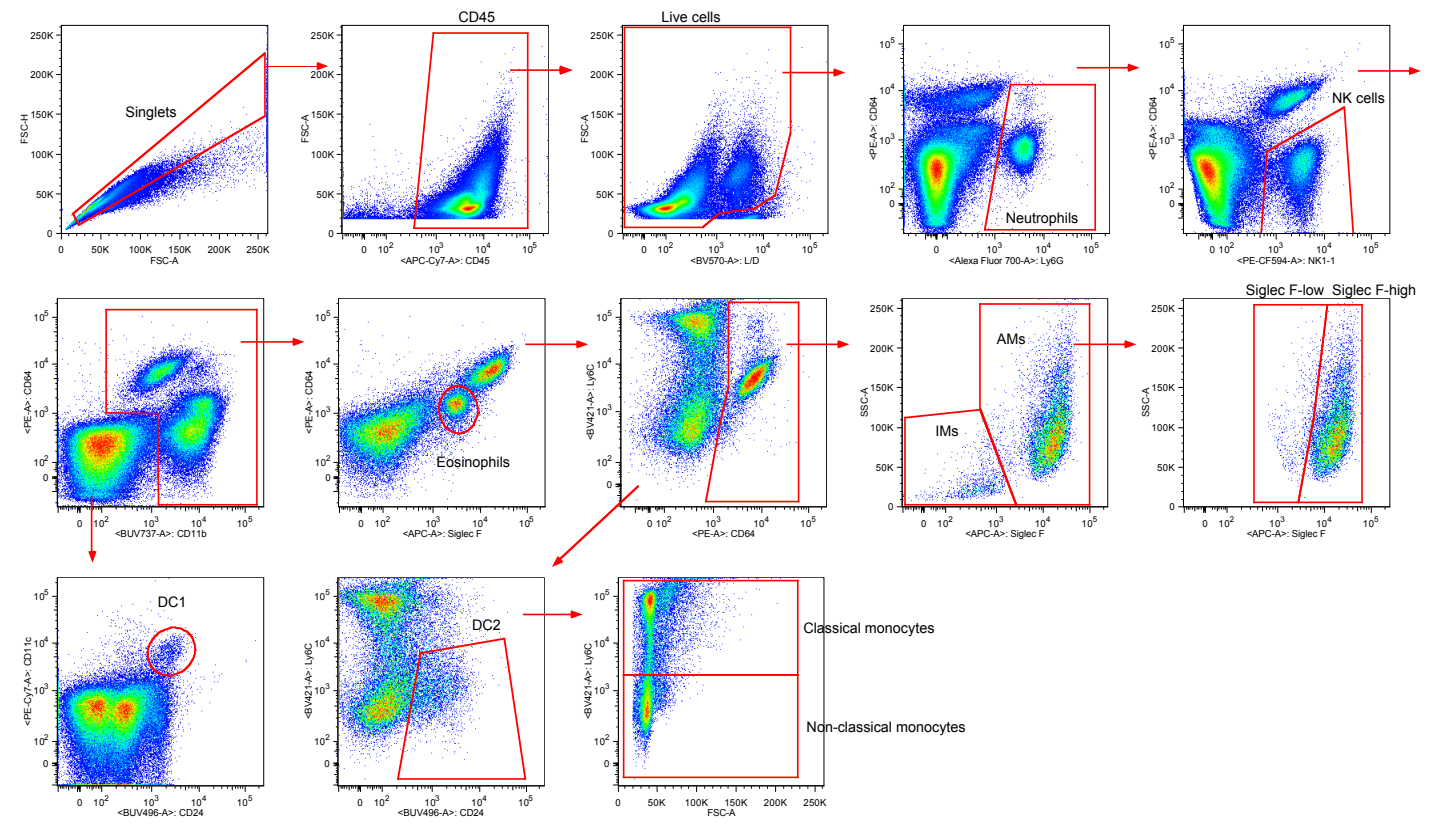

B Gating strategy (lymphoid cells)
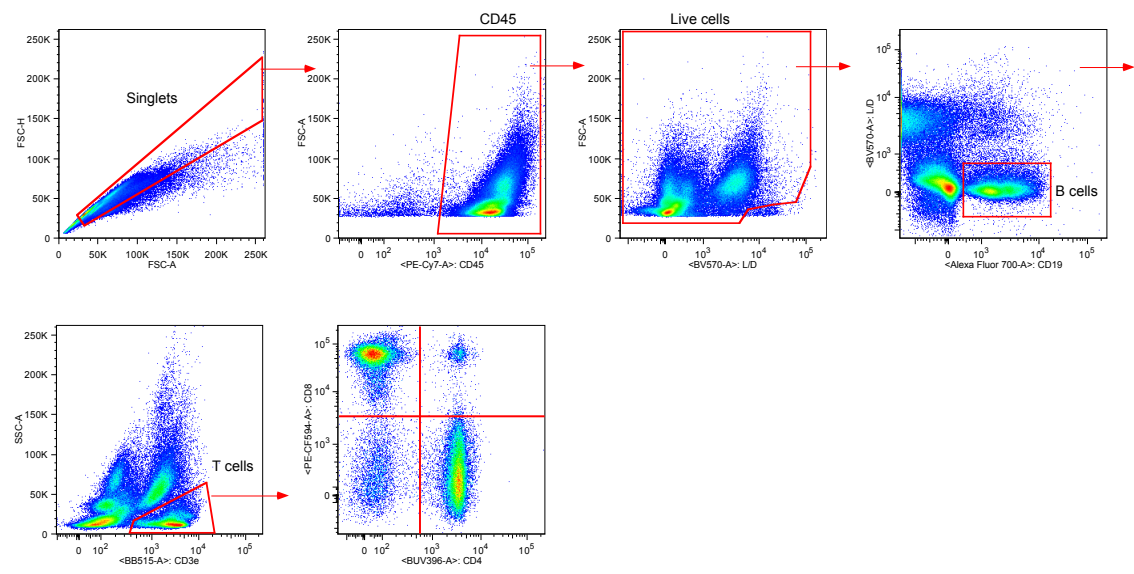

Figure S1. Flow cytometry gating strategy for lung myeloid and lymphoid cell populations. Gating strategy to enumerate and flow cytometry sort (A) myeloid cell populations and (B) lymphoid cell populations.

Representative data from naïve young mouse lung are shown. 

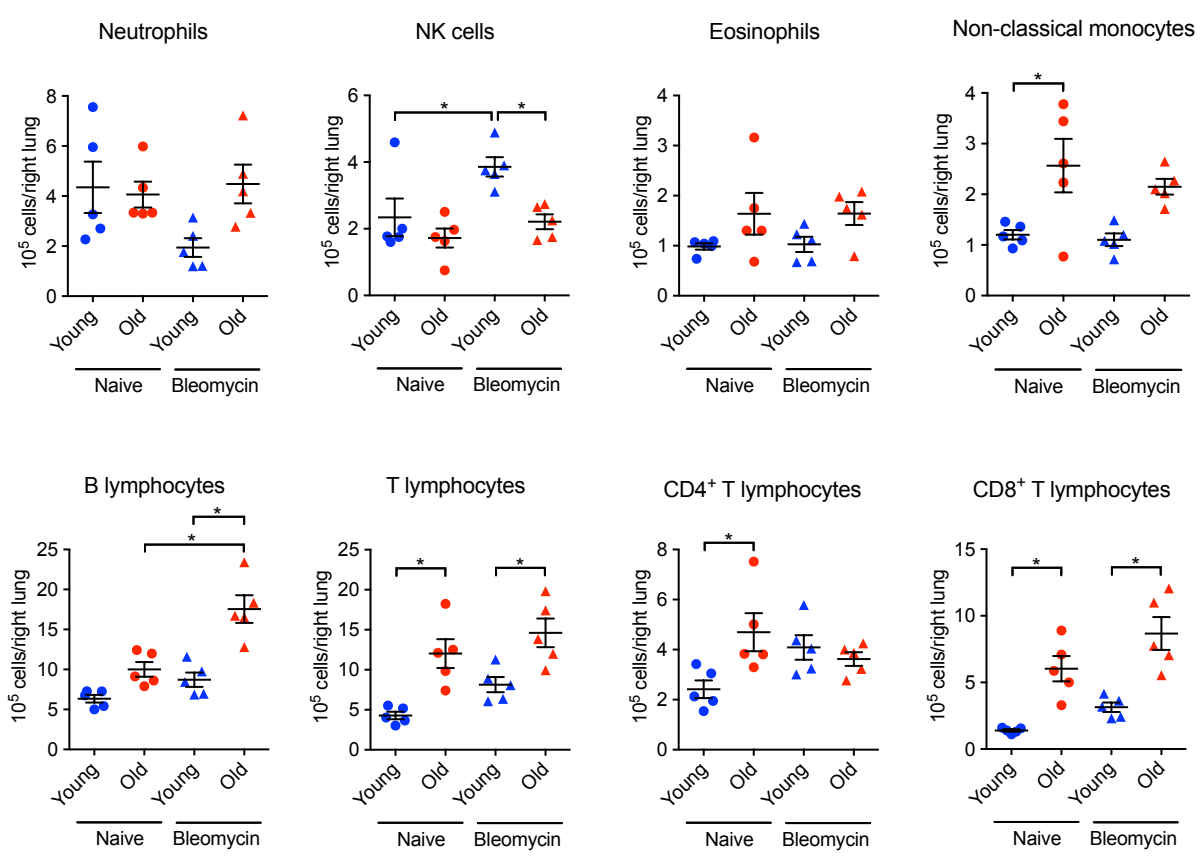

\section{Figure S2. Aging is associated with the changes in the number of lymphoid and myeloid cell populations}

in naïve mice and during lung fibrosis. Myeloid populations including neutrophils, NK cells, eosinophils and non-classical monocytes and lymphoid populations including B cells, $\mathrm{T}$ cells, $\mathrm{CD} 4^{+} \mathrm{T}$ cells and $\mathrm{CD} 8^{+} \mathrm{T}$ cells were enumerated using flow cytometry. Data presented as mean \pm SEM, 5-8 mice per group, one-way ANOVA with Tukey-Kramer test for multiple comparisons. *, $\mathrm{p}<0.05$. 


\section{ISRIB ameliorates pulmonary fibrosis in young and old mice.}

Chronic activation of the integrated stress response (ISR) during aging has been linked to the development of pulmonary fibrosis (33-36). We treated young mice with a small molecule inhibitor of the integrated stress response, ISRIB (25 mg/kg via intraperitoneal injections) or vehicle daily starting at day 7 after instillation of bleomycin, when acute lung injury is largely resolved and active fibrogenesis has begun, and continued until harvest at day 28 (Figure 3A). Treatment with ISRIB resulted in higher lung compliance, lower collagen levels and reduced histologic evidence of fibrosis as measured by blinded scoring of randomly selected lung sections stained with Masson's trichrome (Figure 3C and D). Moreover, young mice treated with ISRIB recovered body weight faster than vehicle-treated mice (Figure 3B). Old mice treated with ISRIB demonstrated attenuated weight loss, increased lung compliance, decreased collagen levels and reduced histologic evidence of fibrosis when compared with vehicle-treated mice (Figure 3E-G). The administration of ISRIB in naïve old or young mice did not affect body weight, lung compliance, collagen levels or induce detectable histologic changes.

We next tested the therapeutic efficacy of ISRIB in a model of non-resolving fibrosis induced by the intratracheal administration of asbestos. Mice were treated with crocidolite asbestos $(100 \mu \mathrm{g} / 50 \mu \mathrm{l})$, and then treated with ISRIB (25 mg/kg via intraperitoneal injections) or vehicle daily beginning 7 days post-instillation. The lungs were harvested and analyzed 28 days after asbestos treatment (Figure S3A). In both young and old mice treatment with ISRIB was associated with reduced evidence of fibrosis as measured by blinded scoring of Masson's trichrome stained lung sections (Figure S3B-C and E-F). As the dynamic range of collagen levels using picrosirius red precipitation (Sircol assay) is too small to detect the differences between the groups, we utilized second harmonic generation microscopy for quantitative analysis of collagen (37). Both young and old mice treated with ISRIB showed a decrease in lung collagen compared to vehicle-treated mice (Figure S3D and $\mathrm{G})$. In addition, young mice treated with ISRIB recovered body weight faster than vehicle-treated mice (Figure S3C and F). Together, these data suggest that ISRIB ameliorates pulmonary fibrosis in both young and old mice in both resolving and non-resolving models. 
bioRxiv preprint doi: https://doi.org/10.1101/2020.02.26.965566; this version posted February 27, 2020. The copyright holder for this preprint (which was not certified by peer review) is the author/funder. All rights reserved. No reuse allowed without permission.

A

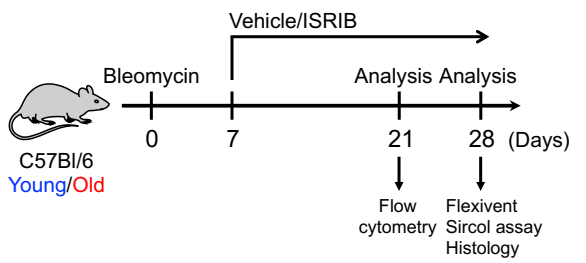

B Young mice

Naive, vehicle

Naive, ISRIB
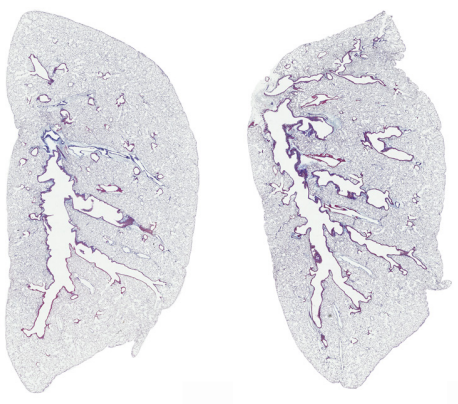

D

Lung compliance

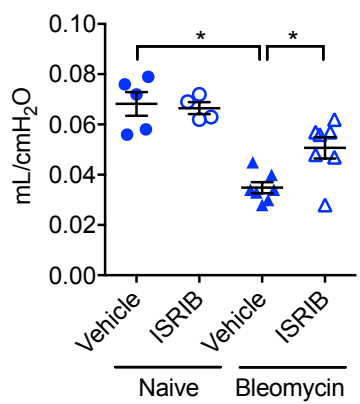

F Old mice

Naive, vehicle

Naive, ISRIB
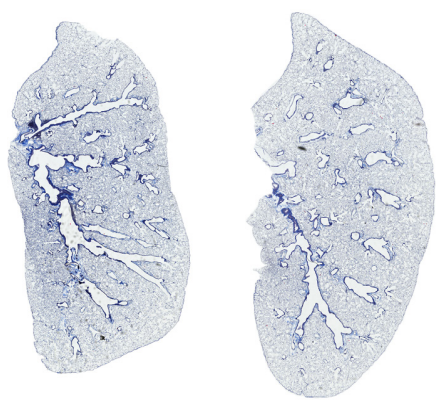

$\mathrm{H}$

Lung compliance

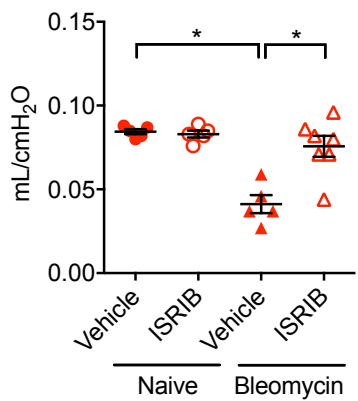

Bleomycin, vehicle

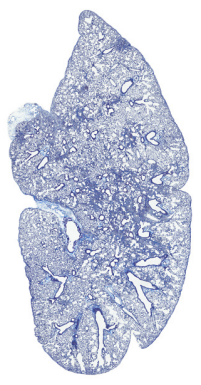

Collagen

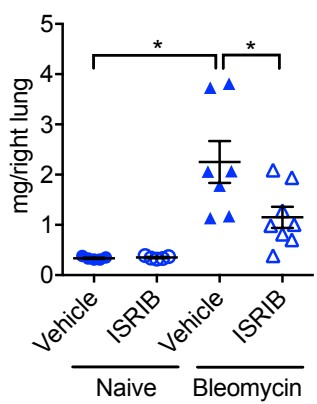

Bleomycin, ISRIB

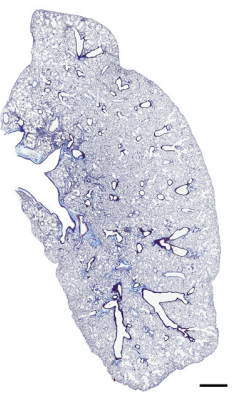

C Fibrosis score

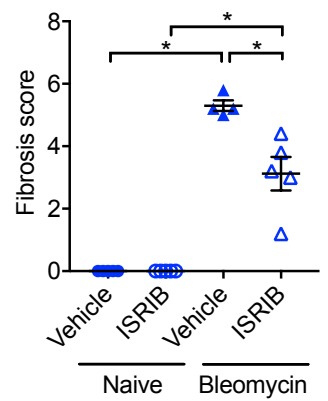

Body weight

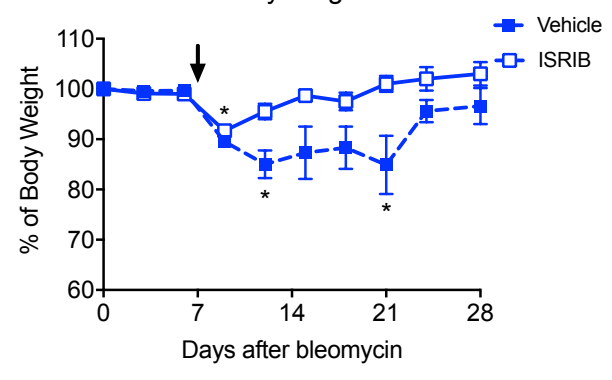

G

Bleomycin, ISRIB

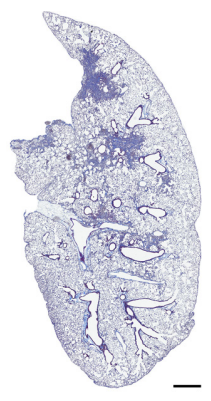

Fibrosis score

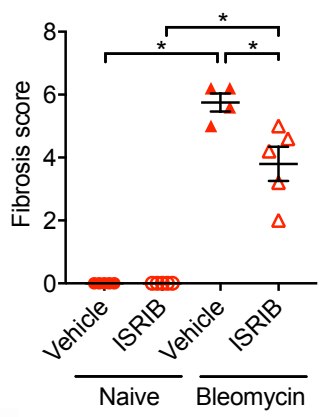

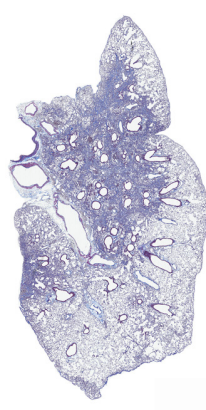

Collagen

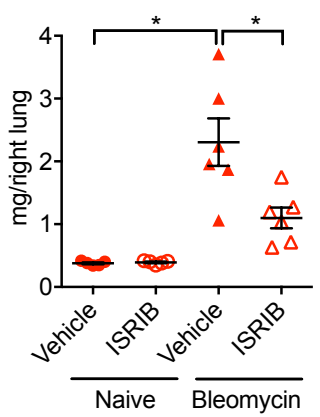

Body weight

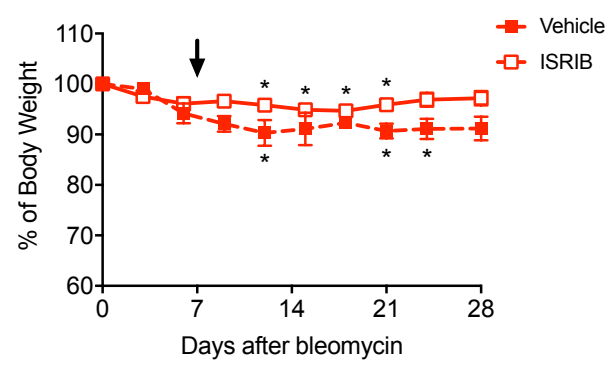


Figure 3. Therapy with ISRIB attenuates bleomycin-induced pulmonary fibrosis in young and old mice.

(A) Schematic of the experimental design. Young (3 months) and old (over 18 months) mice were administered bleomycin (0.025 unit/50 $\mu$, intratracheally) and treated with $25 \mathrm{mg} / \mathrm{kg}$ of ISRIB or vehicle (i.p., daily) beginning at Day 7 when lung injury has resolved and harvested at the indicated time points. (B) Representative images of lung tissue from young naïve mice or bleomycin-treated mice with or without ISRIB on day 28. Masson's trichrome staining. Scale bar $=1 \mathrm{~mm}$. (C-D) Quantification of fibrosis score, lung compliance, and collagen levels from young naïve mice or bleomycin-treated mice with or without ISRIB. (E) The graph shows the body weight of the young mice. 5 mice per each group. Arrow indicates the starting point of ISRIB treatment. (F) Representative images of lung tissue from old naïve mice or bleomycin-treated mice with or without ISRIB on day 28. (G-H) Quantification of fibrosis score, lung compliance, and collagen levels from old naïve mice or bleomycin-treated mice with or without ISRIB. (I) The graph shows the body weight of the old mice. 5 mice per each group. Arrow indicates the starting point of ISRIB treatment. Masson's trichrome staining. Scale bar $=1 \mathrm{~mm}$. Data are shown as mean \pm SEM, 5-7 mice per group. One-way ANOVA with Tukey-Kramer test for multiple comparison or Kruskal-Wallis test with Dunn's multiple comparisons test for comparison of each body weight to baseline (day 0 ). ${ }^{*} \mathrm{p}<0.05$. Representative data from two independent experiments. 
bioRxiv preprint doi: https://doi.org/10.1101/2020.02.26.965566; this version posted February 27, 2020. The copyright holder for this preprint (which was not certified by peer review) is the author/funder. All rights reserved. No reuse allowed without permission.

A

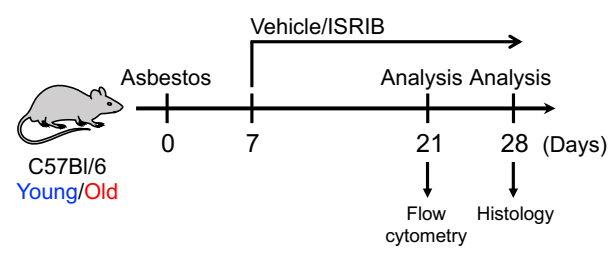

B Young mice

Asbestos, Vehicle

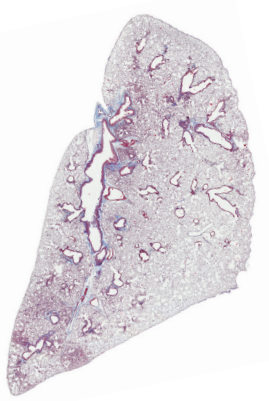

Asbestos, Vehicle

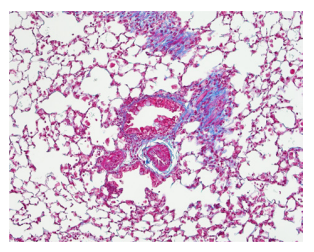

\section{E Old mice}

Asbestos, Vehicle

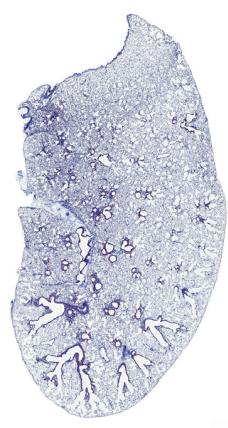

Asbestos, Vehicle

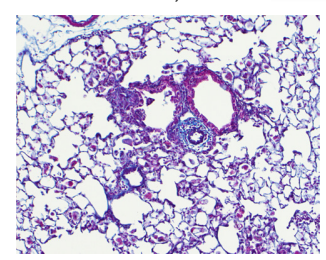

Asbestos, ISRIB

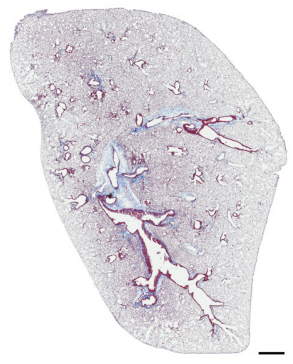

Asbestos, ISRIB

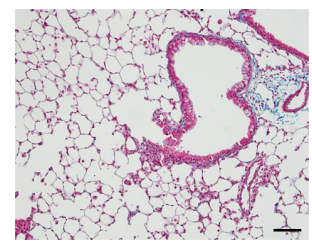

Asbestos, ISRIB

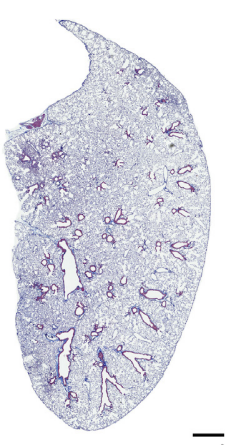

Asbestos, ISRIB

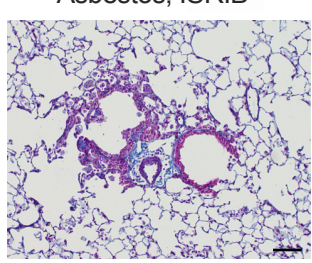

C

Fibrosis score

Collagen
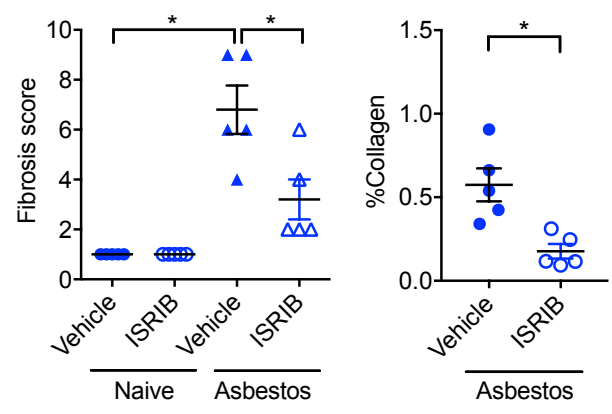

D

Body weight

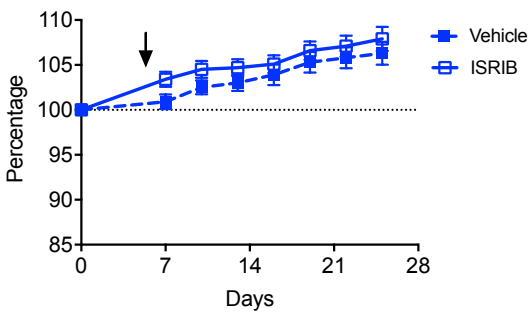

F

Fibrosis score

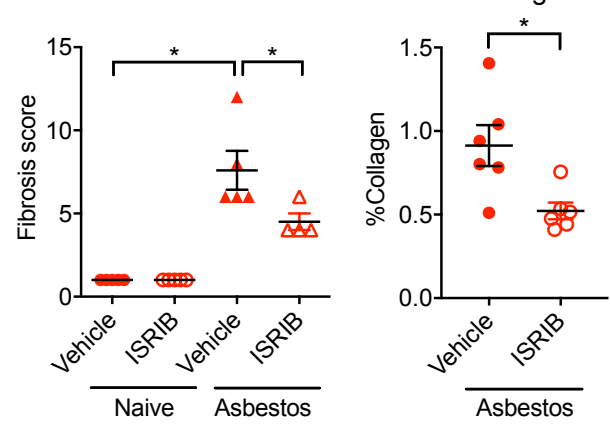

G

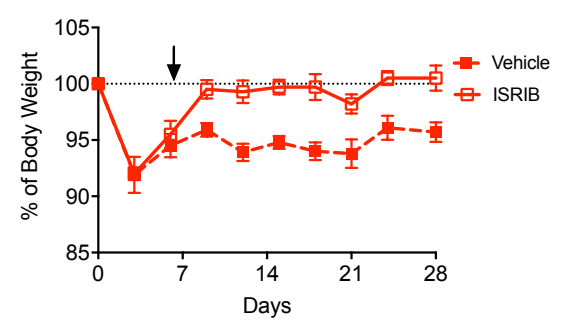




\section{Figure S3. Therapy with ISRIB attenuates asbestos induced pulmonary fibrosis in young and old mice.}

(A) Schematic of the experiment design. Young (3 months) and old (over 18 months) mice were administered crocidolite asbestos $(100 \mu \mathrm{g} / 50 \mu \mathrm{l}$, intratracheally) and treated with $25 \mathrm{mg} / \mathrm{kg}$ of ISRIB or vehicle (i.p., every day) beginning at Day 7 when fibrosis has begun and harvested at the indicated time points. (B, E)

Representative images of lung tissue from naïve mice or asbestos-treated mice with or without ISRIB on day 28 and fibrosis score. Masson's trichrome staining. Scale bar $=1 \mathrm{~mm}$. Representative images in high magnification $(400 \times)$ are also shown. Scale bar $=100 \mu \mathrm{m}$. (C, F) Fibrosis score and the percentage of collagen levels using secondary harmonic generation from naïve mice or asbestos-treated mice with or without ISRIB. (D, G) The graph shows the body weight. Arrow indicates the starting point of ISRIB treatment. Data are shown as mean \pm SEM, 5-7 mice per group. One-way ANOVA with Tukey-Kramer test for multiple comparison, non-parametric analysis with Mann-Whitney test, or Kruskal-Wallis test with Dunn's multiple comparisons test for comparison of each body weight to baseline (day 0$) .{ }^{*} \mathrm{p}<0.05$. Representative data from two independent experiments. 


\section{ISRIB reduces the recruitment of profibrotic monocyte-derived alveolar macrophages to the lung.}

To test whether the amelioration of fibrosis with ISRIB is associated with decreased recruitment of profibrotic monocyte-derived alveolar macrophages, we treated mice with bleomycin and 7 days later started treatment with ISRIB (25 mg/kg, daily via intraperitoneal injections) followed by analysis of myeloid and lymphoid cells in the lung via flow cytometry at day 21. Interestingly, treatment with ISRIB decreased the number of lung monocytes in old mice, but did not affect monocyte levels in young mice (Figure 4A,B). The administration of ISRIB also decreased the number of interstitial macrophages $\left(\mathrm{CD}^{+} 4^{+}\right.$Siglec $\left.\mathrm{F}^{-}\right)$and monocyte-derived alveolar macrophages $\left(\mathrm{CD}^{+} 4^{+}\right.$Siglec $\left.\mathrm{F}^{\text {low }}\right)$, but did not change the number of tissue-resident alveolar macrophages $\left(\mathrm{CD}^{+} 4^{+}\right.$Siglec $\left.\mathrm{F}^{\text {high }}\right)$ in either young or old mice (Figure 4A, B).

We used a genetic lineage tracing system to permanently label circulating monocytes and monocyte-derived alveolar macrophages derived from circulating monocytes $\left(C \times 3 c r 1^{E R C r e} \times z s G r e e n^{L S L}\right)$. Classical monocytes, which are precursors for monocyte-derived alveolar macrophages, have a half-life of $\sim 5-7$ days in mice (38). Therefore, only monocyte-derived alveolar macrophages recruited within this window of time after tamoxifen administration will be labeled with GFP (Figure 5A). We administered tamoxifen simultaneously with bleomycin and started treatment with ISRIB at day 7, harvesting lungs for analysis on days 7, 14 and 21 (Figure 5B). In young mice we found that at all time points nearly $80 \%$ of the Siglec $F^{\text {low }}$ alveolar macrophages were GFP-positive, suggesting that most of the monocyte-derived alveolar macrophages enter the lung after bleomycin injury as a single wave within the first 7 days with negligible recruitment afterwards (Figure 5C and D). In young mice, the number of Siglec $\mathrm{F}^{\text {low }} \mathrm{GFP}^{+}$alveolar macrophages peaked at day 14 and decreased by day 21. Surprisingly, old mice had fewer Siglec Flow $^{\text {GFP }}{ }^{+}$alveolar macrophages at day 7 and 14, but more at day 21. The number of Siglec Flow GFP- alveolar macrophages also increased by day 21, suggesting that persistent epithelial injury in old mice drives the proliferation and ongoing recruitment of monocyte-derived alveolar macrophages (Figure 5E). Treatment with ISRIB did not affect the numbers of tissue-resident alveolar or interstitial macrophages. In contrast, treatment with ISRIB decreased the number of Siglec $\mathrm{F}^{\text {low }} \mathrm{GFP}^{+}$alveolar macrophages at day 14 in young mice and at day 21 in old mice (Figure 5F and G). We have shown that cell surface expression of Siglec F increases as monocyte-derived alveolar macrophages mature (16). Consistently, the expression of Siglec F increased in GFP-positive monocyte-derived alveolar macrophages between days 7 and 21 of bleomycin-induced lung fibrosis in young mice. Interestingly Siglec F levels were higher in GFPpositive macrophages in old mice treated with ISRIB compared with vehicle (Figure S4A and B), suggesting that ISRIB not only reduced recruitment of monocyte-derived alveolar macrophages in old mice but also promoted their maturation. 
A Young mice
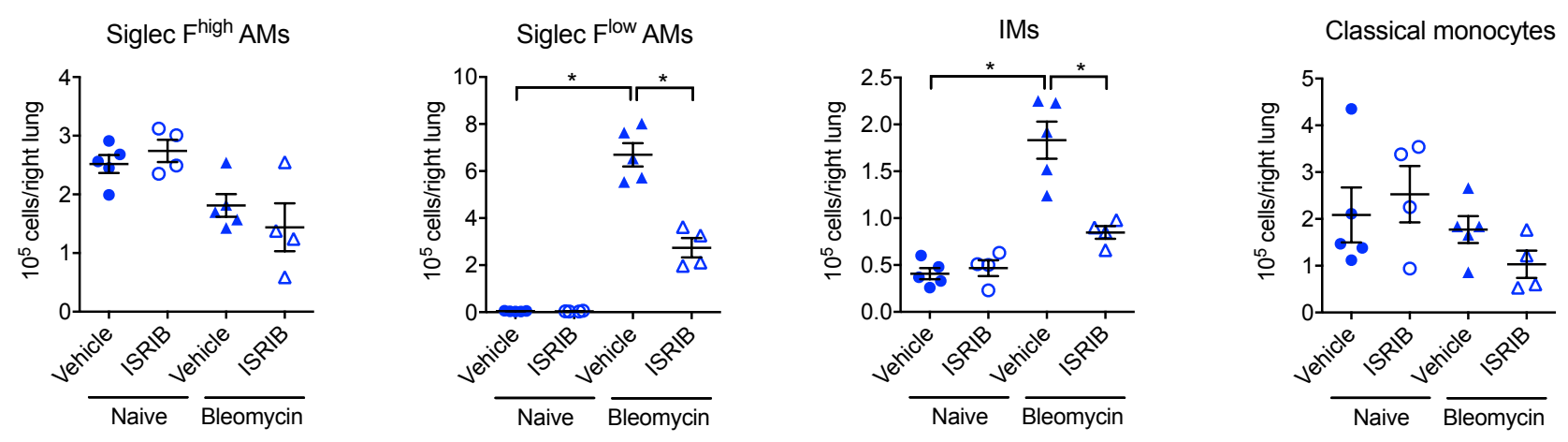

B Old mice
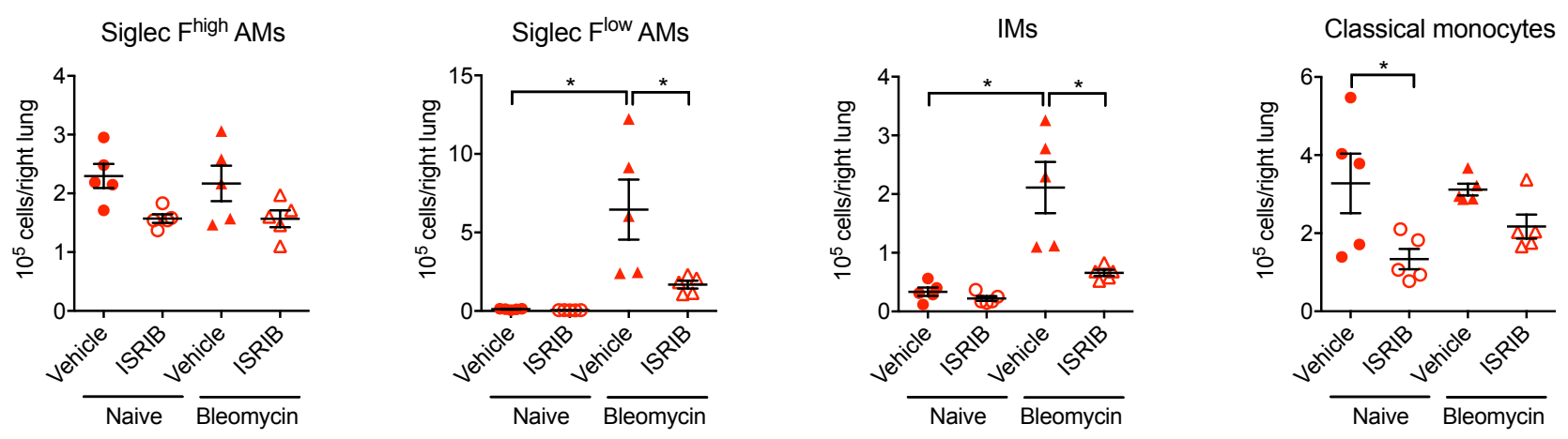

Figure 4. ISRIB reduces the number of monocyte-derived alveolar macrophages in lung fibrosis. Young (3 months) and old (over 18 months) mice were administered 0.025 unit of bleomycin, treated with $25 \mathrm{mg} / \mathrm{kg}$ of ISRIB or vehicle (i.p., every day), and the lungs are analyzed by flow cytometry on day 21 . The number of macrophages and classical monocytes in (A) young and (B) old mice. Data are shown as mean \pm SEM, 5 mice per group. One-way ANOVA with Tukey-Kramer test for multiple comparison. ${ }^{*} \mathrm{p}<0.05$. Representative data from two independent experiments. 
bioRxiv preprint doi: https://doi.org/10.1101/2020.02.26.965566; this version posted February 27, 2020. The copyright holder for this preprint (which was not certified by peer review) is the author/funder. All rights reserved. No reuse allowed without permission.

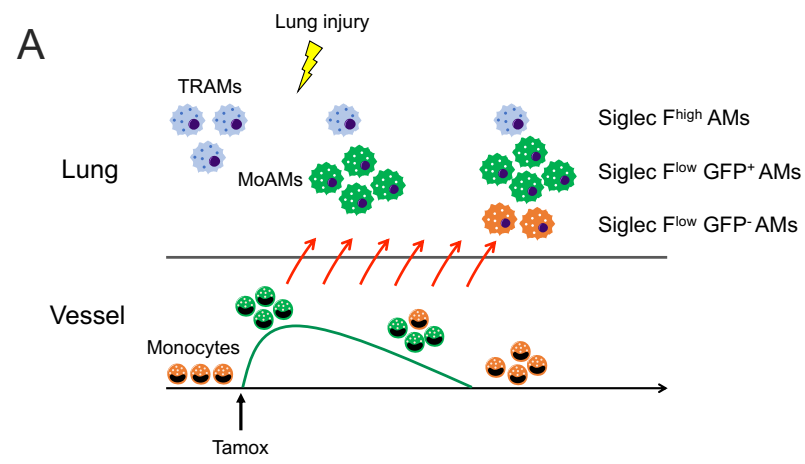

C

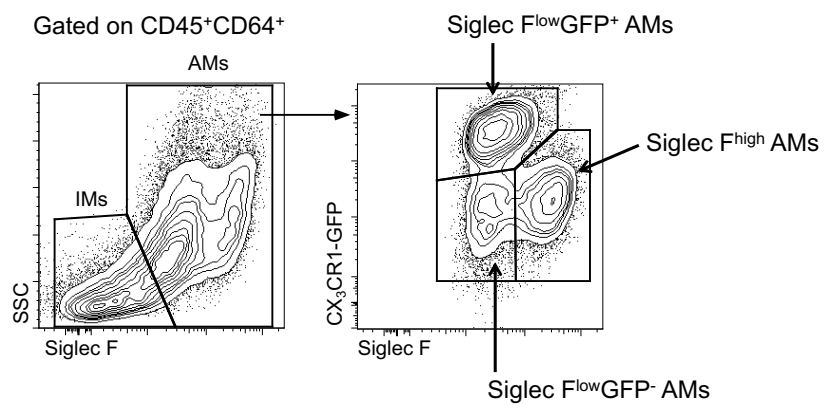

E

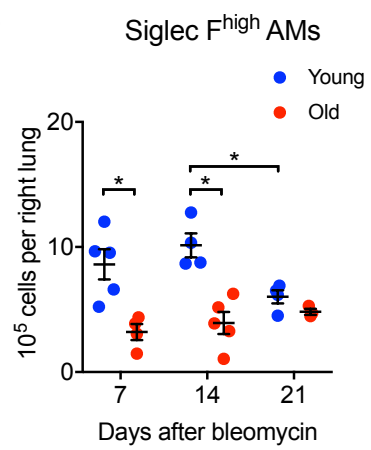

F

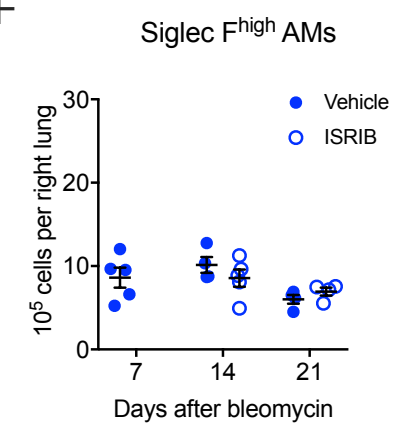

G

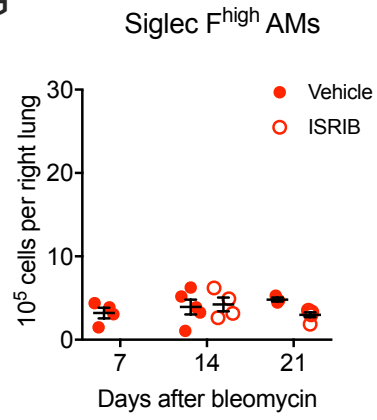

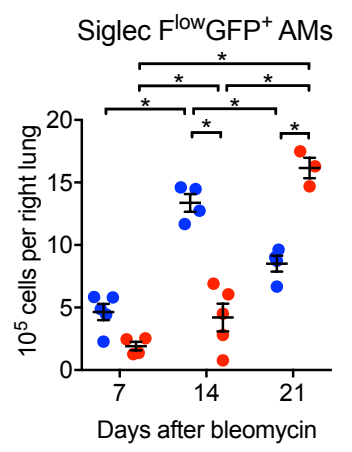

Siglec ${\text { Flow } G F P^{+}}^{\text {AMs }}$

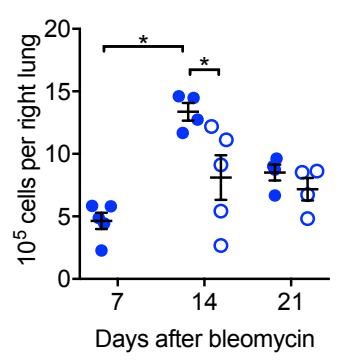

Siglec F low $\mathrm{GP}^{+} \mathrm{AMs}$

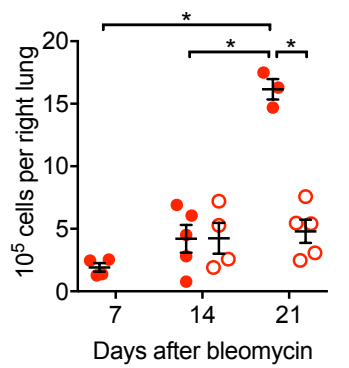

B

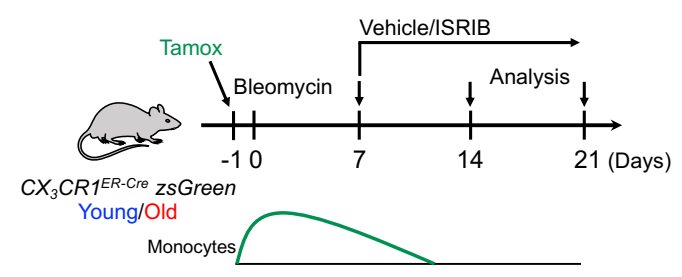

D \%GFP of Siglec Flow AMs
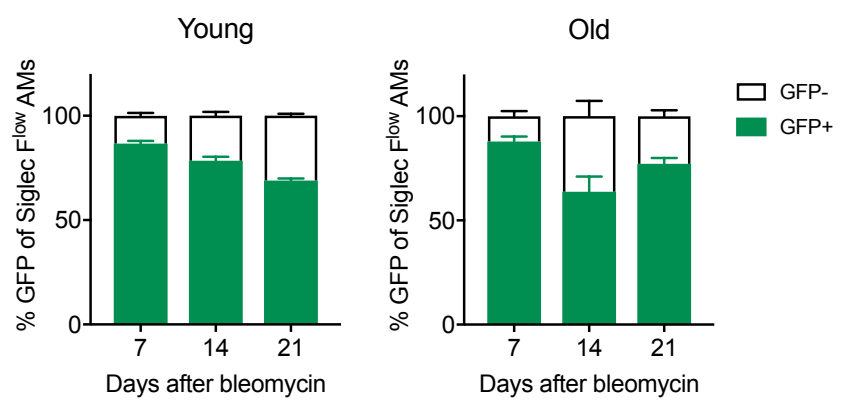

Siglec Flow GFP-AMs

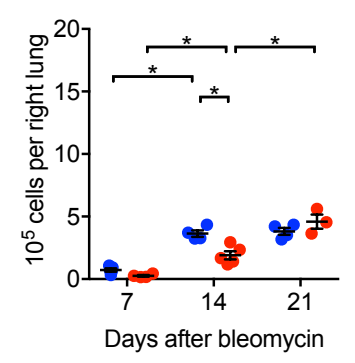

Siglec Flow GFP-AMs

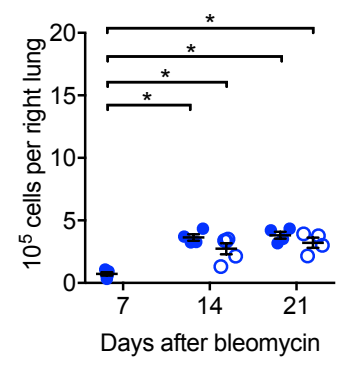

Siglec Flow GFP-AMs

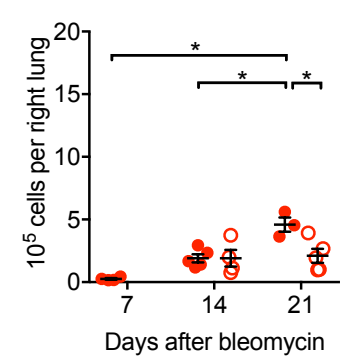




\section{Figure 5. The recruitment kinetics of monocyte-derived alveolar macrophages is different between young} and old mice during pulmonary fibrosis. (A) Schematic of the genetic lineage tracing system using $\mathrm{CX}_{3} C R 1^{\text {ERCre }} \times{ }_{z s}$ Green mice. Circulating monocytes are labeled with GFP after tamoxifen treatment, but tissue-resident alveolar macrophages are not. After lung injury, GFP-labeled monocytes are recruited to the lung and become monocyte-derived alveolar macrophages. GFP-labeled monocytes are partially replaced by newly formed unlabeled cells 7 days later and are completely replaced 21 days later. In this system, we can track the monocyte-derived alveolar macrophages recruited during the initial wave of monocyte influx. (B) Schematic of the experiment design. Young ( 3 months) and old (over 18 months) female $C X_{3} C R 1^{\text {ERCre }} \times z s G r e e n$ mice were used to assess the recruited monocyte-derived alveolar macrophages. The mice were given pulse of tamoxifen (10 mg, p.o.) one day before receiving intratracheal bleomycin. Therapeutic ISRIB or vehicle were administered daily beginning at Day 7 after bleomycin and the lungs were harvested at the indicated times. (C) Flow cytometry gating strategy for macrophage populations. (D) \%GFP of Siglec F ${ }^{\text {low }}$ alveolar macrophages (AMs) during the course of bleomycin model with young and old mice. (E) The number of Siglec Figh AMs, Siglec F $^{\text {low }} \mathrm{GFP}^{+}$AMs and Siglec Flow GFP- AMs in comparison of young with old mice. (F-G) The number of Siglec $F^{\text {high }}$ AMs, Siglec F ${ }^{\text {low }}$ FFP $^{+}$AMs and Siglec Flow GFP- AMs in young and old mice in comparison of vehicle with ISRIB treatment. Data are shown as mean \pm SEM, 3-5 mice per group. One-way ANOVA with TukeyKramer test for multiple comparison. ${ }^{*} \mathrm{p}<0.05$. 
A

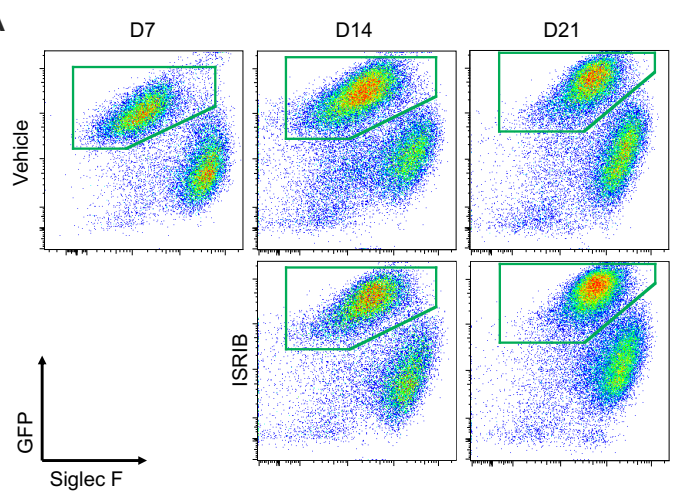

B

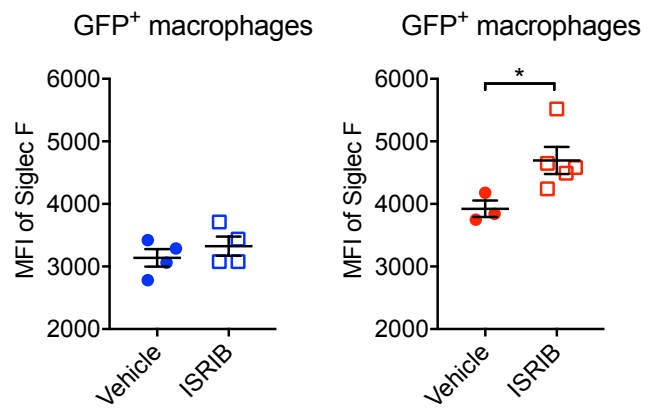

Figure S4. ISRIB promotes the maturation of monocyte-derived alveolar macrophages in old mice during pulmonary fibrosis. (A) Representative flow data gated on $\mathrm{CD} 45^{+} \mathrm{CD} 64^{+}$macrophages. (B) Median fluorescence intensity (MFI) of Siglec F levels on $\mathrm{GFP}^{+}$macrophages in young and old mice 21 days after bleomycin model with and without ISRIB treatment. Data are shown as mean \pm SEM, 3-5 mice per group. Nonparametric analysis with Mann-Whitney test. *p $<0.05$. 


\section{Transcriptomic profiling suggests that ISRIB promotes the differentiation of monocyte-derived alveolar macrophages.}

ISRIB reduced number of lung monocytes in old mice, leaving open the possibility that it can directly target monocytes to reduce the recruitment of monocyte-derived alveolar macrophages. We therefore performed RNA-seq on classical monocytes flow-sorted from the lungs of the young and old naïve mice treated with ISRIB for 7 days. Principal component analysis demonstrated a lack of variance in the data related to either aging or treatment with ISRIB (Figure S5A). A direct pairwise comparison between the groups identified only 2 differentially expressed genes between ISRIB- and vehicle-treated mice irrespective of age (Supplemental Table 1). These results suggest that while treatment with ISRIB alters the number of classical monocytes in the lung, it does not substantially modify their transcriptomic identity.

We then performed RNA-seq on flow-sorted monocyte-derived alveolar macrophages in young and old mice with or without treatment with ISRIB (starting at day 7) in both the bleomycin (harvest day 21, Figure 6A, S5B) and asbestos models of lung fibrosis (harvest day 28, Figure 6B, S5C). Principal component analysis demonstrated that treatment with ISRIB and age were the main sources of variance in the dataset (PC1 29.7\% and PC2 12.2\%, respectively) (Figure S4B). We performed k-means clustering on 1923 differentially expressed genes in the bleomycin dataset (ANOVA-like test, FDR q value <0.05) (Figure 6A, Supplemental Tables S2 and S3). Treatment of bleomycin exposed young and old mice with ISRIB upregulated genes involved in biosynthetic process, positive regulation of proliferation, and phagocytosis (Figure 6A, Cluster 1) and downregulated genes involved in cell cycle, antigen processing, MHC II presentation and innate immune response (Figure 6A, Cluster 2). Genes that were upregulated in monocyte-derived alveolar macrophages from bleomycin-exposed old compared with young mice included those involved in inflammatory response, antigen processing and presentation, and reactive oxygen species production (Figure 6A, Cluster 4). Genes that were specifically upregulated in monocyte-derived alveolar macrophages from old compared with young mice treated with bleomycin followed by ISRIB (Figure 6A, Cluster 5) were enriched for processes characteristic for homeostatic tissue-resident alveolar macrophages such as lipid metabolism and phagocytosis, while downregulated genes (Figure 6A, Cluster 3) were involved in extracellular matrix remodeling, myoblast proliferation, macrophage migration, and chemotaxis.

Our fate-mapping data suggested that after bleomycin, monocyte-derived alveolar macrophages continued to be recruited much longer after bleomycin in old compared with young mice and this prolonged recruitment was prevented by treatment with ISRIB (Figure 5C,D). These differences in the kinetics of recruitment might account for the apparent reduction in fibrotic gene expression in monocyte derived alveolar macrophages from 
ISRIB treated animals in the bleomycin model (Figure 6A, Cluster 3). We therefore performed transcriptomic profiling of tissue-resident and monocyte-derived alveolar macrophages in asbestos-induced pulmonary fibrosis, where we have shown that monocytes are continuously recruited (Figure 6B) (18). Principal component analysis demonstrated macrophage ontogeny and aging as two major sources of variance in the dataset (PC1 54.5\% and PC2 18.6\%, correspondingly, Figure S5C). Importantly, k-means clustering performed on differentially expressed genes (FDR q $>0.05$ in ANOVA-like test) failed to identify genes that differed between asbestos induced monocyte-derived alveolar macrophages in young compared with old mice (Figure 6B, Supplemental Tables S4 and S5). Instead, genes upregulated in monocyte-derived alveolar macrophages compared with tissue resident alveolar macrophages were similar in young and old animals (Figure 6B, Cluster 3) and included genes related to phagocytosis (Mefg8, Clqa, Clqb, C3ar, Itgam, Msrl), extracellular matrix remodeling and fibroblast proliferation (Itgav, Mmp12, Mmp14, Spp1, Thbs 1, Fn1) and monocyte-tomacrophage differentiation and maintenance (Mafb, Bhlhe41, Csflr, Csf1). Similarly, genes upregulated in tissue-resident compared with monocyte-derived alveolar macrophages were similar in young and old animals(Figure 6B, Cluster 2) and included canonical genes expressed in mature alveolar macrophages (Siglecf, Axl, Sirpa, Earl) and were enriched for genes related to positive regulation of RNA biosynthesis and histone modification. In agreement with our previous report, genes upregulated in tissue-resident alveolar macrophages with aging (Figure 6B, Cluster 1) were related to antigen processing and presentation and immune system processes, while genes downregulated in tissue-resident alveolar macrophages from old mice (Figure 6B, Cluster 4) were related to positive regulation of cell cycle (39). 
bioRxiv preprint doi: https://doi.org/10.1101/2020.02.26.965566; this version posted February 27, 2020. The copyright holder for this preprint (which was not certified by peer review) is the author/funder. All rights reserved. No reuse allowed without permission.

A Siglec Flow alveolar macrophages in Bleomycin model

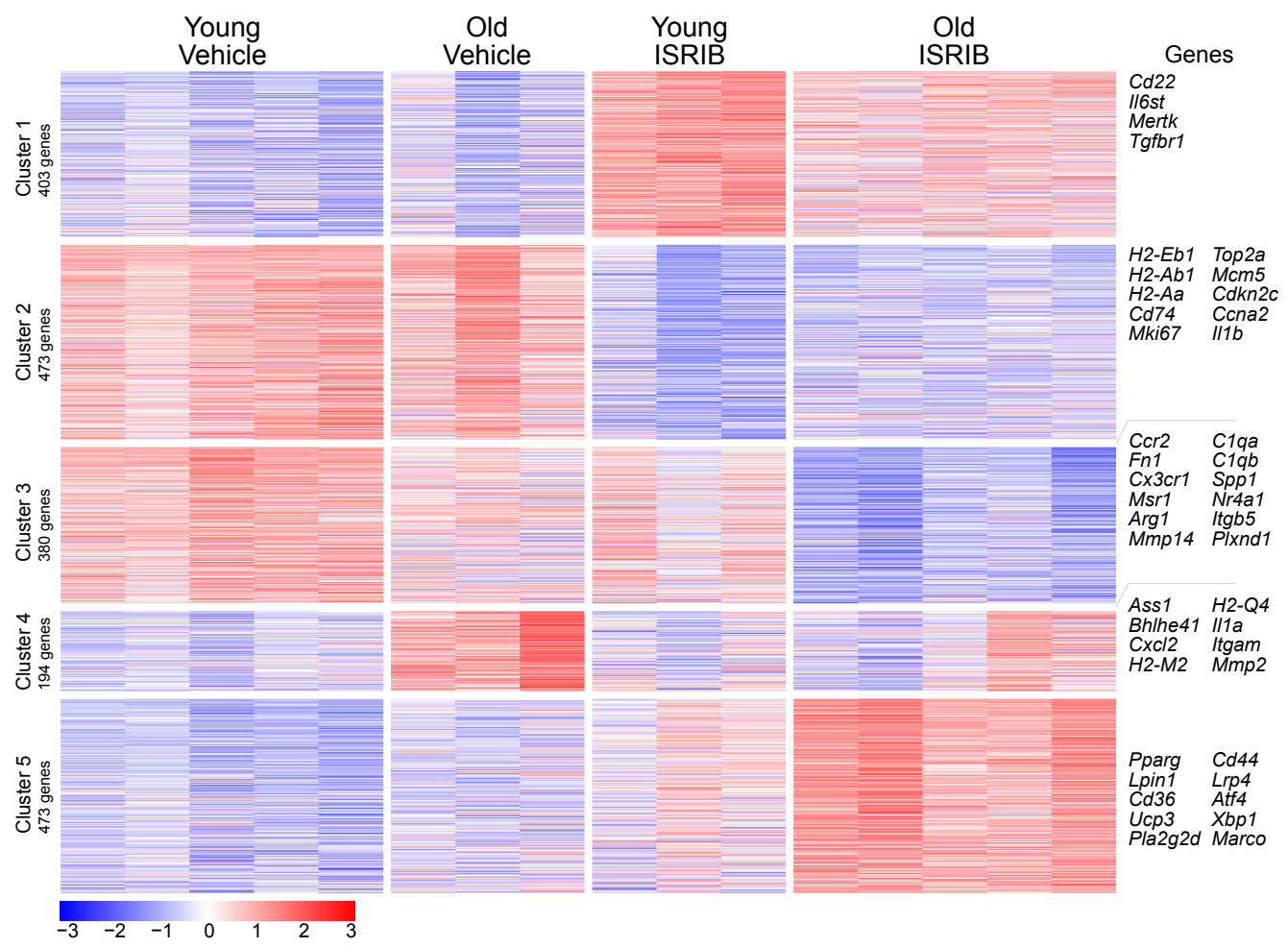

\title{
B Alveolar macrophages in asbestos model
}

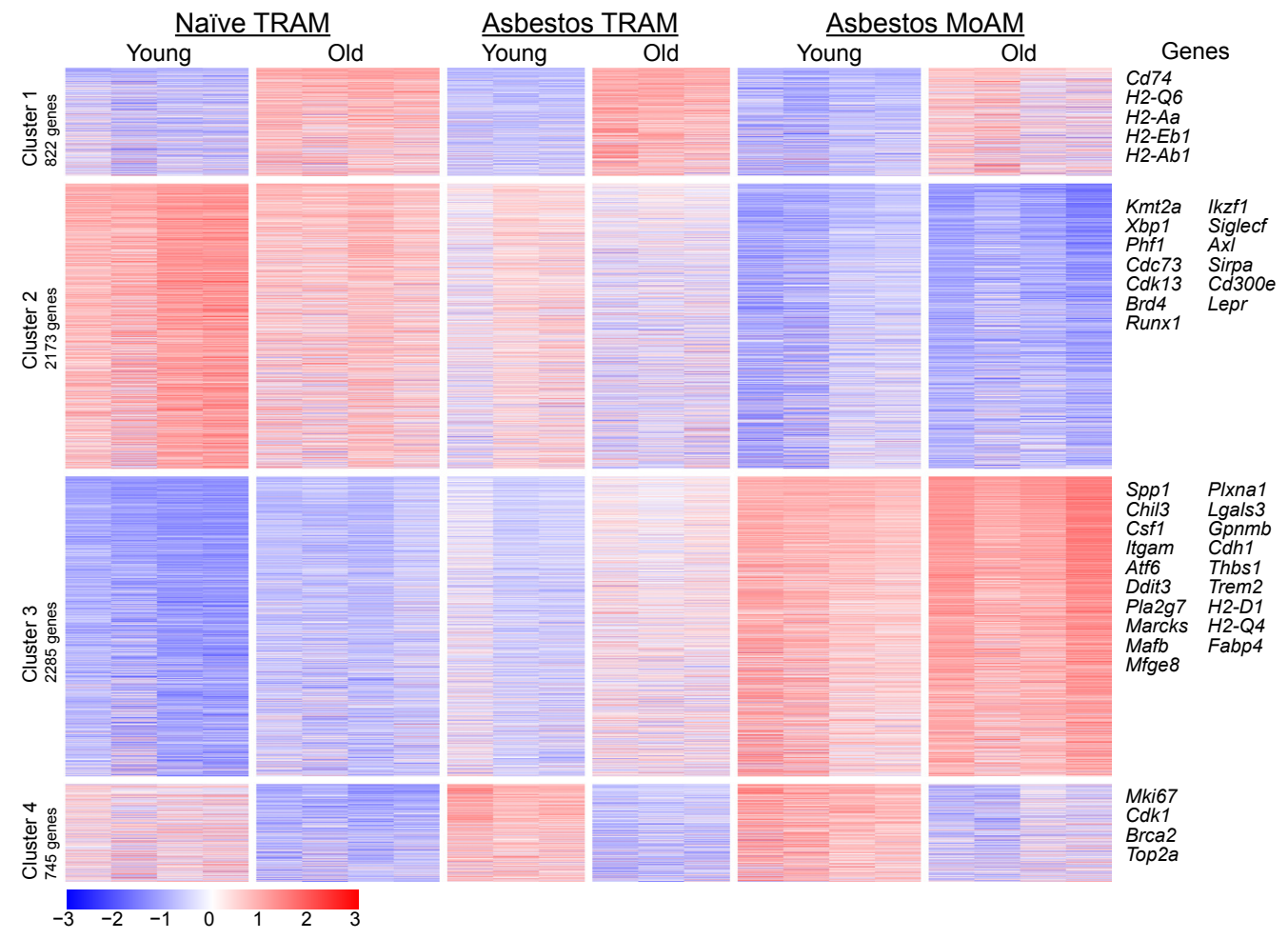

\author{
GO processes \\ Antigen processing and presentation \\ of exogenous peptide antigen \\ Phagocytosis, recognition \\ Immune response \\ Positive regulation of RNA \\ biosynthetic process \\ Hemopoiesis \\ Positive regulation of histon \\ modification \\ Immune response-activating cell \\ surface receptor signaling pathway \\ Response to unfolded protein \\ Extracellular structure organization \\ Antigen processing and presentation \\ of endogenous peptide antigen via \\ MHC class I via ER pathway \\ Fatty acid metabolic process
Response to endoplasmic reticulum \\ stress \\ Apoptotic cell clearance \\ ERAD pathway \\ Mitotic cell cycle phase transition \\ Mitotic spindle assembly checkpoint
}


Figure 6. Transcriptomic profiling suggests that ISRIB promotes the differentiation of monocyte-derived alveolar macrophages. (A) Transcriptomes of sorted monocyte-derived alveolar macrophages in young and old mice bleomycin model with and without ISRIB treatment. k-means clustering of all identified differentiallyexpressed genes (ANOVA-like test on negative binomial generalized log-linear model, FDR q-value $<0.05$ ) revealed clusters of genes especially up- and down-regulated by ISRIB treatment (cluster 1 and 2), genes upand down-regulated by ISRIB especially in old mice (cluster 3 and 5), and genes especially up in old mice and down-regulated by ISRIB (cluster 4). The characteristic GO processes and genes associated with each cluster are shown on the right sides, correspondingly. (B) Transcriptomes of sorted tissue-resident alveolar macrophages (TRAMs) and monocyte-derived alveolar macrophages (MoAMs) in naïve lung and asbestosinduced lung fibrosis. k-means clustering of all identified differentially-expressed genes (ANOVA-like test on negative binomial generalized log-linear model, FDR q-value $<0.05$ ) revealed clusters of genes associated with gene ontogeny (clusters 1 and 4) and genes especially up- and down-regulated in MoAMs in asbestos-induced lung fibrosis (clusters 2 and 3). The characteristic GO processes and genes associated with each cluster are shown on the right sides, correspondingly. 
A Classical monocytes in naive mice

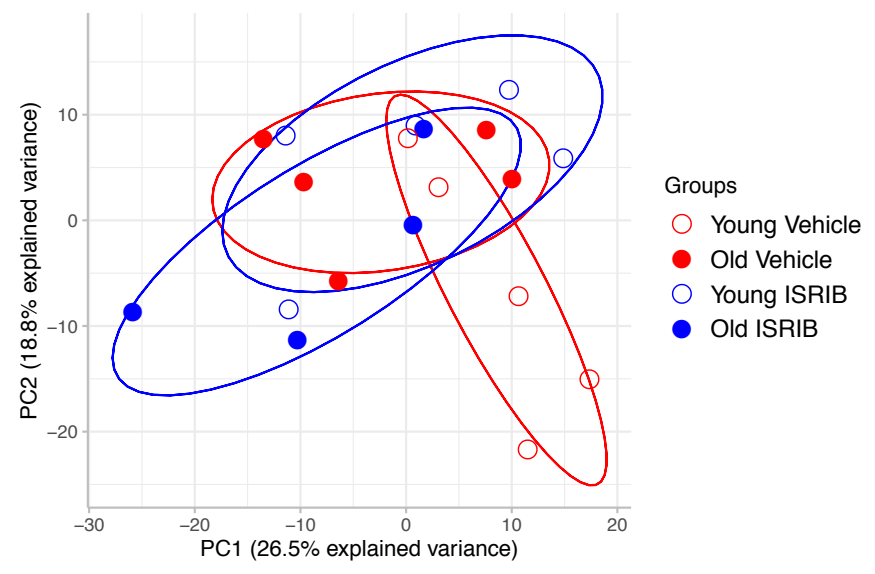

B Siglec Flow alveolar macrophages in bleomycin model

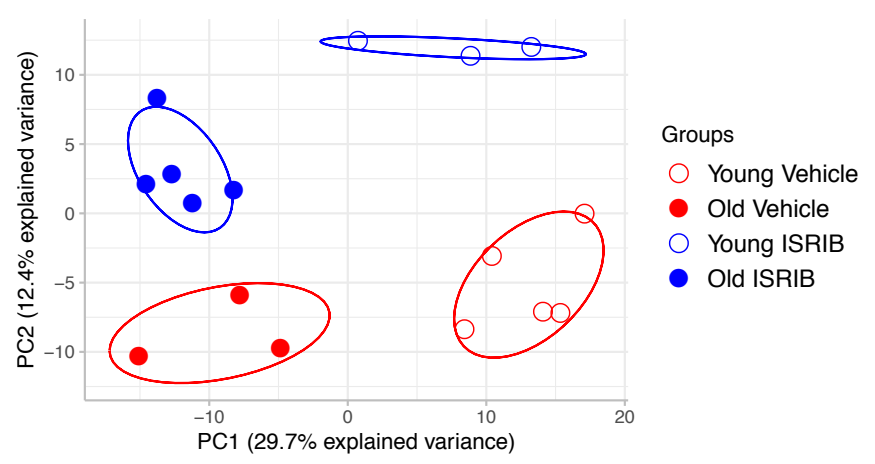

C Alveolar macrophages in asbestos model

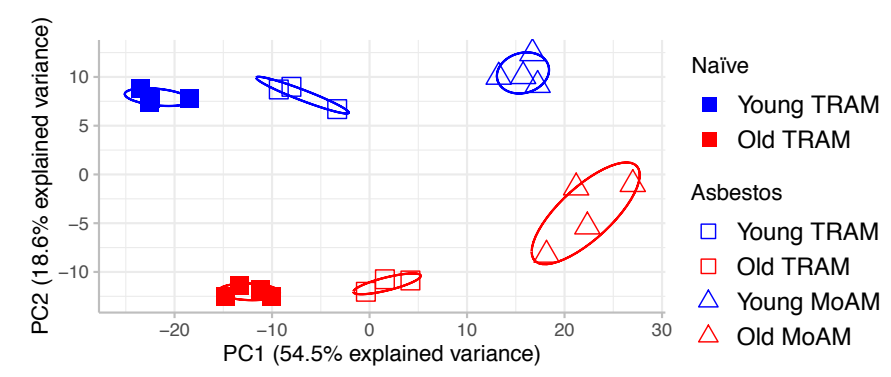

Figure S5. Principal component analysis. (A) Principal component analysis of classical monocytes in young and old naïve mice treated with ISRIB or vehicle did not reveal age- or treatment-correlated sources of variation. (B) Principal component analysis of monocyte-derived alveolar macrophages in young and old mice bleomycin model with and without ISRIB treatment. $\mathrm{PC} 1$ is associated with aging and $\mathrm{PC} 2$ is associated with ISRIB treatment. (C) Principal component analysis of tissue-resident alveolar macrophages (TRAMs) and monocyte-derived alveolar macrophages (MoAMs) in naïve lung and asbestos-induced lung fibrosis. PC1 is associated with pulmonary fibrosis and PC2 is associated with aging. 


\section{A single dose of ISRIB resets proteostasis in the lung epithelium and modulates stress response in epithelial cells.}

Previous studies have demonstrated that even a single dose of ISRIB could dramatically ameliorate ISR-driven diseases $(28,29,40)$. Accordingly, we treated mice with a single dose of ISRIB or vehicle 7 days after administration of bleomycin and assessed severity of fibrosis 21 day later (Figure 7A). Mice treated with ISRIB reduced histologic evidence of fibrosis as measured by blinded scoring of randomly selected lung sections stained with Masson's trichrome, improved lung compliance and decreased collagen levels (Figure 7B and C). In addition, we asked whether prophylactic administration of ISRIB is sufficient to ameliorate bleomycininduced pulmonary fibrosis. We treated mice with a single dose of ISRIB or vehicle immediately prior to bleomycin instillation. Mice treated with ISRIB exhibited less severe fibrosis (Figure 7B and C). Moreover, mice treated with ISRIB lost significantly less weight than vehicle-treated mice (Figure 7D).

These data suggest that ISRIB ameliorates pulmonary fibrosis by modulating signals upstream of monocytederived alveolar macrophage recruitment. As intratracheal bleomycin and asbestos primarily impact the lung epithelium, we treated mice with a single dose of ISRIB or vehicle and 24 hours later performed transcriptomic profiling on flow-sorted alveolar epithelial type II cells: 100 genes were downregulated and 109 genes were upregulated (FDR q < 0.05). The upregulated genes included genes critical to protein folding (Hspala, Hspalb, Hsph1, Bag3, Dnajb1 and others), while downregulated genes were enriched for biological processes related to cell death and cell differentiation (Figure 7E, Supplemental Table S6, S7). These data suggested that a single dose of ISRIB was sufficient to induce preemptive upregulation of chaperones in epithelial cells, rendering them more resistant to subsequent stress and apoptosis. We and others have shown that genetic strategies that prevent apoptosis reduce the severity of pulmonary fibrosis in response to bleomycin or forced overexpression of TGF- $\beta(41,42)$. We administered ISRIB 6 days after the intratracheal instillation of bleomycin and quantified the number of apoptotic cells 24 hours later using TUNEL assay. Treatment with ISRIB significantly reduced the number of TUNEL-positive cells (Figure 7F and G). 
bioRxiv preprint doi: https://doi.org/10.1101/2020.02.26.965566; this version posted February 27, 2020. The copyright holder for this preprint (which was not certified by peer review) is the author/funder. All rights reserved. No reuse allowed without permission.

A

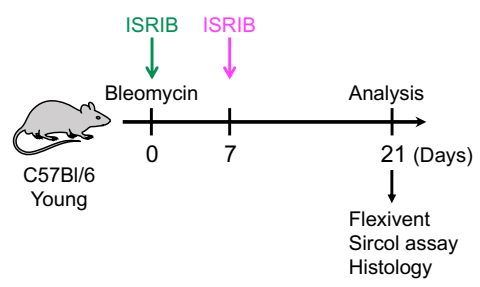

B
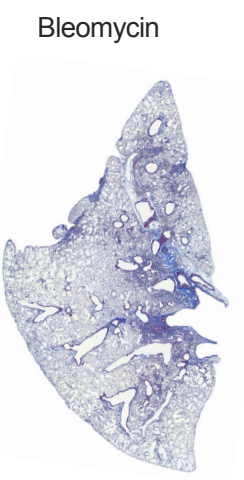

C
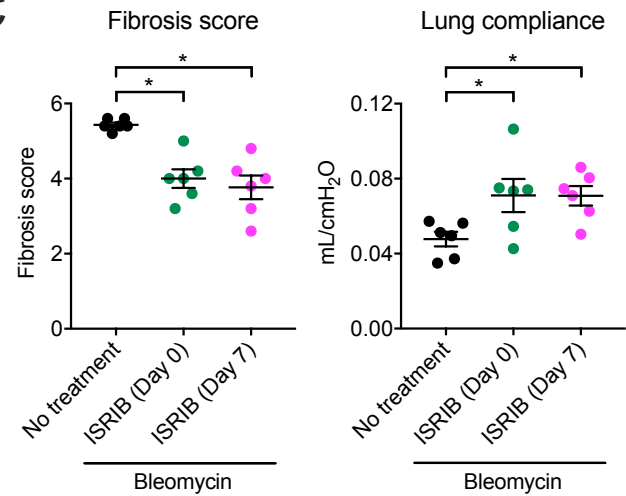

E

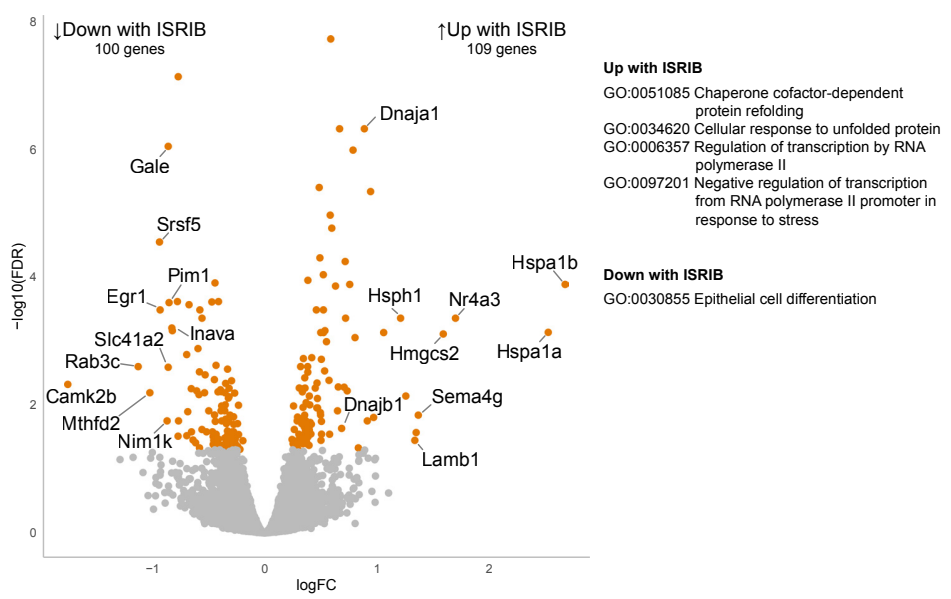

\section{F}

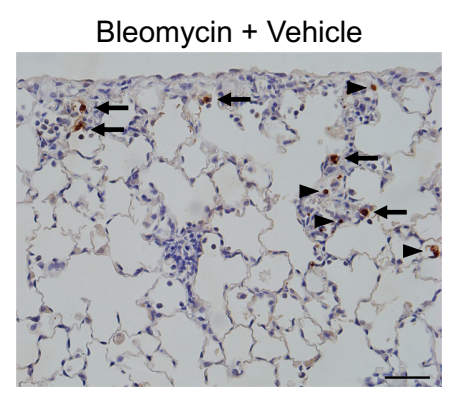

Collagen levels

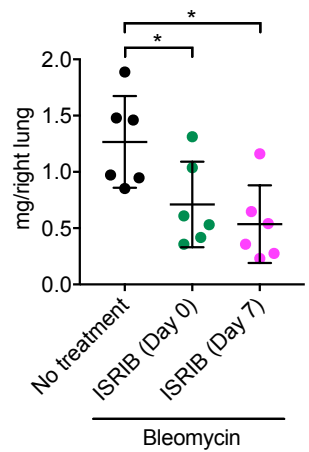

Bleomycin
G

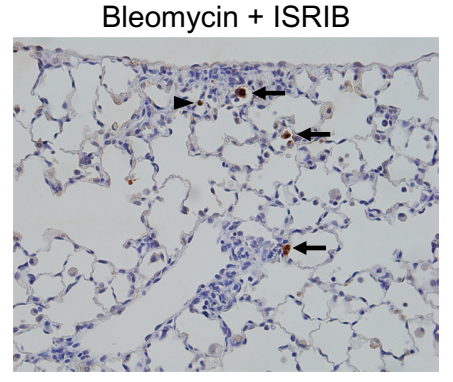

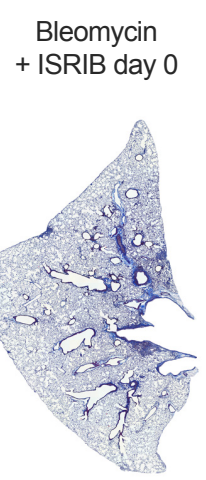

Bleomycin

+ ISRIB day 7

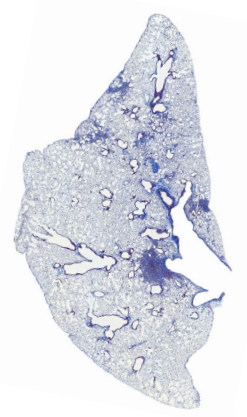

Body weight

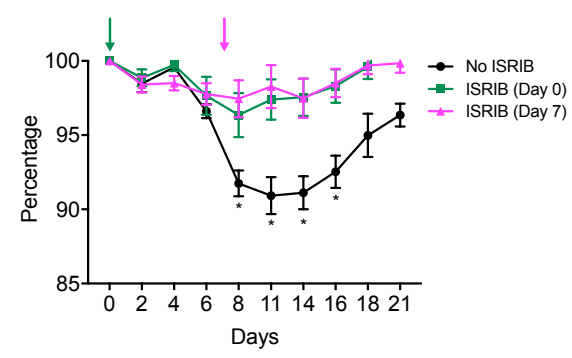

TUNEL $^{+}$cells

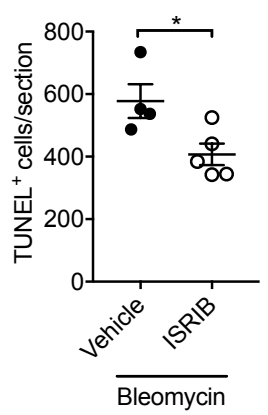


Figure 7. ISRIB ameliorates fibrosis by modulating stress response in epithelial cells. (A) Schematic of the experiment outline. Young ( 3 months) mice were administered 0.025 unit of bleomycin and treated with 25 $\mathrm{mg} / \mathrm{kg}$ of ISRIB according to the time line. (B) Representative histologic images in bleomycin model (Masson's trichrome, Scale bar $=1 \mathrm{~mm}$ ). (C) Quantification of fibrosis score using Ashcroft score, lung compliance using Flexivent, and soluble collagen in lung homogenates in bleomycin model. (D) Body weight in bleomycin model with single dose of ISRIB treatment. Arrow indicates the point of ISRIB treatment. (E) Volcano plot showing number of differentially expressed genes in alveolar epithelial cells in naïve young mice 24 hours after vehicle or ISRIB treatment (FDR q-value $<0.05)$. Characteristic GO processes are shown on the right $(\mathrm{p}$-value $<0.05)$. (F) Representative figures of lung tissue with TUNEL staining from bleomycin-treated young mice at day 7 that were treated with single dose of ISRIB or vehicle 24 hours before. Arrows indicate TUNEL-positive epithelial cells and triangle arrows indicate TUNEL-positive immune cells. Scale bar $=100 \mu \mathrm{m}$. (G) The number of TUNEL-positive cells per section. Data are shown as mean \pm SEM, 4-5 mice per group. One-way ANOVA with Tukey-Kramer test for multiple comparison, non-parametric analysis with Mann-Whitney test, or KruskalWallis test with Dunn's multiple comparisons test for comparison of each body weight to baseline $($ day 0$)$. ${ }^{*} p<$ 0.05 . 


\section{DISCUSSION}

Our data add to an emerging literature elucidating the multicellular nature of pulmonary fibrosis. We found that a small molecule inhibitor of the integrated stress response - ISRIB - resets proteostasis in the alveolar epithelium thereby reducing the recruitment of pathogenic monocyte-derived alveolar macrophages. We and others have shown that after recruitment these monocyte-derived alveolar macrophages form stable, perturbation-resistant circuits that drive fibroblast proliferation and matrix production (16-18). Over time these fibroblasts may gain the ability to maintain proliferation and matrix production independent of macrophages, leading to localized areas of unchecked fibrosis $(43,44)$.

We observed that old mice developed more severe pulmonary fibrosis in response to both bleomycin and asbestos. We used a genetic lineage tracing system that allowed us to measure the dynamics of monocytederived alveolar macrophage recruitment over the course of resolving (bleomycin)- and non-resolving (asbestos)-induced fibrosis in young and aged mice. In young mice exposed to bleomycin, monocyte-derived alveolar macrophages were recruited as a single wave of cells confined to the first 14 days after injury. In contrast, more monocyte-derived alveolar macrophages were recruited to the lungs of old mice, and recruitment was ongoing even 21 days after the injury. Consistently, the recruitment of monocyte-derived alveolar macrophages was enhanced at all time points in old compared with young mice after asbestos exposure. Transcriptomic profiling suggested minimal differences in profibrotic gene expression of monocyte-derived alveolar macrophages in old compared with young mice and mice treated with ISRIB. Instead, an apparent increase in fibrotic gene expression in monocyte-derived alveolar macrophages from old mice after bleomycin was attributable to ongoing recruitment of immature cells.

Combined, these lineage tracing and transcriptomic studies suggest that age-related changes in the number and transcriptomic identity of monocyte-derived alveolar macrophages during fibrosis are not cell-autonomous. Instead, these changes represent responses to the aging lung microenvironment. This is in agreement with our previous finding in normally aged mice that orthotopic heterochronic adoptive transfer of tissue-resident alveolar macrophages from young into old mice results in a change of transcriptomic identity that reflects the age of the recipient (39). Because ISRIB reduced the recruitment of monocyte-derived alveolar macrophages to the lungs of old and young mice without affecting their transcriptomic identity, these findings suggest that ISRIB targets the lung epithelium to reduce fibrosis in these models. Indeed, even a single dose of ISRIB given to naïve mice was sufficient to induce the expression of Hspla and Hsplb, key members of HSP70 chaperone family, and their co-chaperone with a known anti-apoptotic function - Bag3 (45). Consistently, the administration of ISRIB reduced the levels of apoptosis during bleomycin-induced lung injury. Whether ISRIB 
reduced the recruitment of monocyte-derived alveolar macrophages by reducing epithelial susceptibility to bleomycin- or asbestos-induced injury, promoting epithelial repair or enhancing the age-related loss of homeostatic signals necessary for alveolar macrophage maturation, is not known.

ISRIB is a small molecule developed by Carmela Sidrauski and colleagues in Peter Walter's laboratory that targets the integrated stress response (ISR) (29). The ISR is activated in response to environmental or genetic stressors that threaten the proteome including ER stress (via PERK), viral infection (via PKR), amino acid deprivation (via GCN2) or heme deprivation (via HRI) (12). These kinases all phosphorylate eIF2 $\alpha$, a component of eukaryotic initiation factor 2 (eIF2), which is required to initiate translation on AUG start codons as a part of eIF2-GTP-Met-tRNA ternary complex. During translation initiation GTP is hydrolyzed to GDP, and a dedicated guanine nucleotide exchange factor for eIF2 (eIF2B) is needed to catalyze GDP-to-GTP exchange and reactivate eIF2 (44). Phosphorylation of eIF2 $\alpha$ causes it to bind tightly to eIF2B precluding binding of the unphosphorylated protein and preventing binding to GTP (46). This inhibits bulk translation but enhances translation of proteins with small upstream open reading frames. Important among these is ATF4, a transcription factor that drives the expression of proteins that protect the proteome including chaperones and metabolic enzymes among others (47). ISRIB enhances guanine exchange factor activity of eIF2B in the presence of phosphorylated eIF2 $\alpha$, relieving both translational inhibition and gene expression induced by ATF4 (29). Like many stress responses, the activation of ATF4 exhibits hormesis; short-term activation is cytoprotective while longer-term or very high level activation is detrimental to the cell (48). Indeed, ATF4 induces the transcription of CHOP, which induces apoptosis upon reaching a threshold level (49). Therefore, ISRIB might function to reduce ISR and promote an adaptive response and survival short-term, while being detrimental during the chronic stress (50).

Our finding that ISRIB attenuates bleomycin- and asbestos-induced fibrosis in young and old mice adds to a growing body of literature showing dramatic effects of ISRIB in age-related disease. A single dose of ISRIB has been shown to improve cognitive function in normal mice and in mice after traumatic brain injury $(28,29,51)$, prevent neurodegeneration in murine prion disease (52), improve glucose tolerance and diabetes induced liver injury in rats (53), prevent postnatal hearing loss in a genetic mouse model (54), reduce neuropathic pain in a murine model of diabetic neuropathy (55), alleviate dwarfism in a murine model of chondrodysplasia (56), slow the growth of metastatic prostate cancer (57), improve survival of lymphoblast cell lines from patients with vanishing white matter disease (58), and alleviate the social deficit and elevated anxiety-like behavior in a murine model of these neuropsychiatric disorders (59). Interestingly, while ISRIB improved memory in normal animals, it did not do so in two murine models of Alzheimer's disease $(60,61)$. 
The finding that ISRIB targets the epithelium to prevent lung fibrosis is consistent with genetic evidence implicating proteostatic stress in the initiation of pulmonary fibrosis (62). For example, a mutation in the MUC5B promoter associated with fibrosis results in enhanced expression of an abundant mucin secreted by lung epithelial cells (25). A mutation in SFTPC, the expression of which is restricted to alveolar type II cells, encodes a misfolded protein and expression of this mutant in alveolar type II cells from mice is sufficient to induce spontaneous pulmonary fibrosis $(20,21,24)$. Loss of function in genes associated with the HermanskyPudlak syndrome disrupt intracellular protein transport and investigators have localized the effect of these mutants to the lung epithelium in animal models $(22,23,63)$. While genes involved in the maintenance of telomere length are ubiquitously expressed, loss of function mutations in these genes targeted to alveolar type II cells induce spontaneous or more severe fibrosis in animal models $(64,65)$. Consistent with these findings, genetic and pharmacologic strategies to inhibit ER stress have been reported to reduce bleomycin-induced fibrosis $(34,36,66)$.

Our study has several limitations. First, while we showed transcriptomic changes in the lung epithelium, reduced apoptosis and reduced numbers of recruited monocyte-derived alveolar macrophages in response to administration of ISRIB during the development of the fibrosis, ISRIB might also have effects on lung repair, during the resolution phase. Second, our finding that age-related increase of the number of lung monocytes was ameliorated by ISRIB treatment suggest it might have effects in the bone marrow or other organs that might secondarily protect the epithelium. Finally, ISRIB both relieves translational inhibition and inhibits the expression of ATF4-mediated gene expression. Whether one or both of these is required for the reduction in monocyte-derived macrophage recruitment and prevention of fibrosis will require additional study.

In conclusion, we show that ISRIB, a small molecule inhibitor of the ISR, can attenuate resolving fibrosis induced by bleomycin and non-resolving fibrosis induced by asbestos fibers in both young and old mice. ISRIB resets proteostasis to enhance the stress resistance of the alveolar epithelium, reducing the recruitment of monocyte-derived alveolar macrophages. ISRIB shows promise as part of a combined strategy targeting epithelial proteostasis, pathogenic monocyte-derived alveolar macrophages and proliferative fibroblasts to treat pulmonary fibrosis. 


\section{MATERIALS AND METHODS}

\section{Key Resources Table}

\begin{tabular}{|c|c|c|}
\hline REAGENT or RESOURCE & SOURCE & IDENTIFIER \\
\hline \multicolumn{3}{|l|}{ Antibodies } \\
\hline Rat anti-mouse I-A/I-E (MHC II), BUV395 & BD Biosciences & Cat\# 743876, RRID:AB 2741827 \\
\hline Rat anti-mouse CD24, BUV496 & BD Biosciences & Cat\#564664, RRID:AB_2716853 \\
\hline Rat anti-mouse CD11b, BUV737 & BD Biosciences & Cat\#564443, RRID:AB_2738811 \\
\hline Rat anti-mouse Ly-6C, eFluor 450 & eBioscience & Cat\#48-5932-82, RRID:AB_10805519 \\
\hline Mouse anti-mouse CD64, PE & BioLegend & Cat\#139303, RRID:AB $10 \overline{6} 13467$ \\
\hline Mouse anti-mouse NK-1.1, PE-CF594 & BD Biosciences & Cat\#562864, RRID:AB_2737850 \\
\hline Hamster anti-mouse CD11c, PE-Cy7 & BD Biosciences & Cat\#558079, RRID:AB 647251 \\
\hline Rat anti-mouse Siglec F, Alexa Fluor 647 & BD Biosciences & Cat\#562680, RRID:AB_2687570 \\
\hline Rat anti-mouse CD45, APC-Cy7 & BD Biosciences & Cat\#557659, RRID:AB_396774 \\
\hline Rat anti-mouse Ly-6G, Alexa Fluor 700 & BD Biosciences & Cat\#561236, RRID:AB_10611860 \\
\hline Armenian hamster anti-mouse CD3e, FITC & eBioscience & Cat\#11-0031-82, RRID:AB_464882 \\
\hline Rat anti-mouse CD25, PE & eBioscience & Cat\#12-0251-82, RRID:AB 465607 \\
\hline Rat anti-mouse CD8a, PE & BioLegend & Cat\#100762, RRID:AB_2564027 \\
\hline Rat anti-mouse CD62L, APC-eFluor 780 & eBioscience & Cat\#47-0621-82, RRID:AB_1603256 \\
\hline Rat anti-mouse CD19, Alexa Fluor 700 & eBioscience & Cat\#56-0193-82, RRID:AB_837083 \\
\hline Rat anti-mouse CD4, BUV395 & BD Biosciences & Cat\#563790, RRID:AB_2738426 \\
\hline Rat anti-mouse CD44, BUV737 & BD Biosciences & Cat\#564392, RRID:AB 2738785 \\
\hline \multicolumn{3}{|l|}{ Chemicals, Peptides, and Recombinant Proteins } \\
\hline Bleomycin & Fresenius Kabi & Cat\#0703-3154-01 \\
\hline Asbestos (UICC crocidolite) & \multicolumn{2}{|c|}{ Provided by US Environmental Protection Agency } \\
\hline ISRIB & $\begin{array}{l}\text { AdooQ } \\
\text { BioScience }\end{array}$ & Cat\#A14302 \\
\hline \multicolumn{3}{|l|}{ Critical Commercial Assays } \\
\hline EdU & $\begin{array}{l}\text { ThermoFisher } \\
\text { Scientific }\end{array}$ & Cat $\# C 10044$ \\
\hline $\begin{array}{l}\text { Click-iT Plus EdU Alexa Fluor } 647 \text { Flow } \\
\text { Cytometry Assay Kit }\end{array}$ & $\begin{array}{l}\text { ThermoFisher } \\
\text { Scientific }\end{array}$ & Cat\#C10634 \\
\hline PicoPure RNA Isolation Kit & $\begin{array}{l}\text { ThermoFisher } \\
\text { Scientific }\end{array}$ & Cat\#KIT0204 \\
\hline AllPrep DNA/RNA Mini Kit & Qiagen & Cat $\# 80204$ \\
\hline NEBNext RNA Ultra I kit & $\begin{array}{l}\text { New England } \\
\text { Biolabs }\end{array}$ & Cat\#E7530L \\
\hline polyA mRNA isolation module & $\begin{array}{l}\text { New England } \\
\text { Biolabs }\end{array}$ & Cat\#E7490S \\
\hline $\begin{array}{l}\text { SMARTer Stranded Total RNA-Seq Kit v2 - } \\
\text { Pico Input Mammalian kit }\end{array}$ & Takara Bio & Cat\#634413 \\
\hline \multicolumn{3}{|l|}{ Deposited Data } \\
\hline & & \\
\hline \multicolumn{3}{|l|}{ Experimental Models: Organisms/Strains } \\
\hline Mouse: C57BL/6J (8-12 weeks old) & $\begin{array}{l}\text { The Jackson } \\
\text { Laboratory }\end{array}$ & JAX: 000664 \\
\hline
\end{tabular}




\begin{tabular}{|c|c|c|}
\hline Mouse: C57BL/6J (over 18 months old) & $\begin{array}{l}\text { National Institute } \\
\text { of Aging }\end{array}$ & \\
\hline Mouse: $C \times 3 c r 1^{E R C r e}$ & $\begin{array}{l}\text { The Jackson } \\
\text { Laboratory }\end{array}$ & JAX: 020940 \\
\hline Mouse: ZsGreen & $\begin{array}{l}\text { The Jackson } \\
\text { Laboratory }\end{array}$ & JAX: 007906 \\
\hline \multicolumn{3}{|l|}{ Software and Algorithms } \\
\hline ImageJ & $\begin{array}{l}\text { Schneider et al., } \\
2012\end{array}$ & https://imagej.nih.gov/ij/ \\
\hline bcl2fastq 2.19 .1 & & $\begin{array}{l}\text { https://support.illumina.com/sequencin } \\
\text { g/sequencing_software/bcl2fastq- } \\
\text { conversion-software.html }\end{array}$ \\
\hline FastQC & & $\begin{array}{l}\text { https://www.bioinformatics.babraham.a } \\
\text { c.uk/projects/fastqc/ }\end{array}$ \\
\hline Trimmomatic 0.36 & & $\begin{array}{l}\text { http://www.usadellab.org } / \mathrm{cms} / \text { ?page }=\text { tr } \\
\text { immomatic }\end{array}$ \\
\hline STAR 2.6.0 & $\begin{array}{l}\text { Dobin et al., } \\
2013\end{array}$ & https://github.com/alexdobin/STAR \\
\hline htseq-count 0.11 .2 & $\begin{array}{l}\text { Anders et al., } \\
2014\end{array}$ & https://htseq.readthedocs.io/en/latest/ \\
\hline edgeR 3.28.0 & Robinson, 2010 & \\
\hline \multicolumn{3}{|l|}{ Other } \\
\hline & & \\
\hline
\end{tabular}

\section{Methods Details}

Mice: All experimental protocols were approved by the Institutional Animal Care and Use Committee at Northwestern University (Chicago, IL, USA). All strains including wild-type mice are bred and housed at a barrier- and specific pathogen-free facility at the Center for Comparative Medicine at Northwestern University. Eight to twelve weeks old mice were used as young mice and over eighteen months old mice were used as old mice for all experiments. The C57BL/6J mice were obtained from Jackson laboratories (Jax stock 000664). Old mice were obtained from National Institute of Aging. The Cx3cr ${ }^{E R C r e}$ mice (67) and ZsGreen (68) mice were obtained from Jackson laboratories (Jax stocks 020940 and 007906, correspondingly).

Bleomycin- and asbestos-induced lung fibrosis and drug administration: Mice were anesthetized and intubated followed by intratracheally instillation of bleomycin ( 0.025 unit in $50 \mu 1$ PBS) or crocidolite asbestos fibers (100 $\mu \mathrm{g}$ in $50 \mu \mathrm{PBS}$ ) to induce lung-fibrosis, as previously described $(16,18)$. Lungs were harvested at indicated time points for flow cytometry, RNA sequencing, and histopathology. ISRIB (AdooQ BioScience, A14302) was first reconstituted in DMSO to the concentration $4 \mathrm{mg} / \mathrm{ml}$, and then further diluted using phosphate buffered saline to the final concentration $0.3125 \mathrm{mg} / \mathrm{ml}$ (52). ISRIB was administered via 
intraperitoneal injection, either via single or daily injections, as indicated in the text and figure legends, at the dose $2.5 \mathrm{mg} / \mathrm{kg}$ in a final volume of $200 \mathrm{ul}$. Control animals were treated using vehicle.

Tissue preparation, flow cytometry and sorting: Tissue preparation for flow cytometry analysis and cell sorting was performed as previously described $(16,18)$, with modifications. Briefly, mice were euthanized and their lungs were perfused through the right ventricle with $10 \mathrm{ml}$ of HBSS. The lungs were removed and infiltrated with $2 \mathrm{mg} / \mathrm{ml}$ collagenase D (Roche, Indianapolis, Indiana) and $0.2 \mathrm{mg} / \mathrm{ml}$ DNase I (Roche, Indianapolis, Indiana) dissolved in HBSS with $\mathrm{Ca}^{2+}$ and $\mathrm{Mg}^{2+}$, using syringe with $30 \mathrm{G}$ needle. Lungs were chopped with scissors, tissue was transferred into C-tubes (Miltenyi Biotech, Auburn, California, 130-096-334), and processed in a GentleMACS dissociator (Miltenyi Biotech, Auburn, California) using program m_lung_01, followed by incubation for 30 minutes at $37^{\circ} \mathrm{C}$ with gentle agitation, followed by program m_lung_02. The resulting single-cell suspension was filtered through a $40 \mu \mathrm{m}$ nylon cell strainer. The cells were incubated with anti-mouse CD45 microbeads (Miltenyi Biotech, Auburn, California,130-052-301) and CD45 cells were collected using the MultiMACS Cell24 Separator (Miltenyi Biotech, Auburn, California), using possel protocol. Automated cell counting was performed using Nexcelom K2 Cellometer with AO/PI reagent. Cells were stained with fixable viability dye eFluor 506 (eBioscience, Waltham, Massachusetts), incubated with FcBlock (BD Biosciences, San Jose, California), and stained with a mixture of fluorochrome-conjugated antibodies, as listed above. Click-it reaction for Alexa Fluor 647 labelling were performed using the Click-iT Plus EdU Alexa Fluor 647 Flow Cytometry Assay Kit (Thermo Fisher Scientific, C10634). Single color controls were prepared using BD CompBeads (BD Biosciences, San Jose, California) and Arc beads (Invitrogen, Waltham, Massachusetts). Flow cytometry and cell sorting were performed at the Northwestern University Robert H. Lurie Comprehensive Cancer Center Flow Cytometry Core facility (Chicago, Illinois). Data were acquired on a custom BD FACSymphony instrument using BD FACSDiva software (BD Biosciences, San Jose, California). Compensation and analysis were performed using FlowJo software (TreeStar). Each cell population was identified using sequential gating strategy (Figure S2). The percentage of cells in the live/singlets gate was multiplied by the number of live cells using Cellometer K2 Image cytometer to obtain cell counts. Cell sorting was performed using BD FACSAria III instrument using 100 um nozzle and 40 psi pressure.

Bulk RNA-seq: RNA was isolated using Arcturus PicoPure RNA Isolation Kit (Thermo Fisher Scientific, catalog number KIT0204) for experiments in Figures 6A, 7C or AllPrep DNA/RNA Mini Kit (Qiagen, catalog number 80204) for experiment in Figure 6B. RNA quality was assessed using TapeStation 4200 (Agilent, Santa Clara, California), only samples with RNA integrity number over 7 were used for library preparation. Library construction for RNA-seq was performed using NEBNext RNA Ultra I kit (New England Biolabs, catalog number E7530L) with polyA mRNA isolation module (New England Biolabs, catalog number E7490S) from 
30ng (Figure 6B), 3ng (Figure 6A) or 50ng (Figure 7C) of RNA. Libraries were assessed for quality (TapeStation 4200, Agilent, Santa Clara, California) and then sequenced on NextSeq 500 instrument (Illumina, San Diego, California). FASTQ files were generated using bcl2fast (version 2.19.1), followed by quality control using FastQC, trimming using Trimmomatic (version 0.36) and mapping to mm10 version of the mouse genome with STAR (version 2.6.0). Counts were generated using htseq-count (HTSeq framework, version 0.11.2). Differential gene expression was performed using edgeR (version 3.28.0). Raw data are available from GEO: GSE145295, GSE145590, GSE145771. Detailed R code used for analysis is available at https://github.com/NUPulmonary/Watanabe2020.

Histopathology and immunohistochemistry: After euthanasia and perfusion, trachea was cannulated with Luer syringe stub blunt needle and mouse lungs were inflated with 4\% paraformaldehyde at $15 \mathrm{~cm}_{2} \mathrm{O}$ column pressure. Left lung was fixed in 4\% paraformaldehyde for 24 hours, dehydrated and embedded in paraffin. For histopathology $4 \mu \mathrm{m}$ thick sections were prepared. Hematoxylin and eosin staining and Masson's trichrome staining were performed for the analysis of fibrosis scoring. TUNEL assay was performed for the analysis of apoptotic cells. Immunohistochemistry was performed at Northwestern University Mouse Histology and Phenotyping Laboratory Core facility (Chicago, Illinois).

Fibrosis scores and lung collagen determination: Fibrosis scores were performed from Masson's trichromestained specimens in a blinded manner, in accordance with modified Ashcroft score for bleomycin (69) and the code set by pathology standard for asbestosis (70). Collagen levels were determined using Sircol assay, as described previously $(16,18)$. For collagen levels using second harmonic generation, the deparaffinized mouse lung tissue were imaged on Nikon A1R-MP Multiphoton Microscope at Northwestern University Nikon Cell Imaging Facility (Chicago, Illinois).

Statistical analysis: Statistical tests and tools for each analysis are explicitly described with the results or detailed in figure legends. 


\section{LIST OF SUPPLEMENTAL TABLES}

Supplemental table S1: Number of differentially-expressed genes between young/old ISRIB/vehicle samples of sorted monocytes. Related to Figure S6A.

Supplemental table S2: List of differentially-expressed genes, their clusters and statistics from Figure 6A. Supplemental table S3: List of GO biological processes for each cluster in Figure 6A.

Supplemental table S4: List of differentially-expressed genes, their clusters and statistics from Figure 6B. Supplemental table S5: List of GO biological processes for each cluster in Figure 6B.

Supplemental table S6: List of differentially-expressed genes and their statistics from Figure 7C.

Supplemental table S7: List of GO biological processes for up- and down-regulated genes in Figure 7C. 


\section{REFERENCES}

1. Lederer DJ, Martinez FJ. Idiopathic pulmonary fibrosis. N Engl J Med 2018;378(19):1811-1823.

2. Richeldi L, Collard HR, Jones MG. Idiopathic pulmonary fibrosis. Lancet 2017;389(10082):1941-1952.

3. Thannickal VJ, Murthy M, Balch WE, Chandel NS, Meiners S, Eickelberg O, Selman M, Pardo A, White ES, Levy BD, et al. Blue journal conference. Aging and susceptibility to lung disease. Am J Respir Crit Care Med 2015;191(3):261-269.

4. Raghu G, Weycker D, Edelsberg J, Bradford WZ, Oster G. Incidence and prevalence of idiopathic pulmonary fibrosis. American journal of respiratory and critical care medicine 2006;174(7):810-816.

5. Raghu G, Chen SY, Yeh WS, Maroni B, Li Q, Lee YC, Collard HR. Idiopathic pulmonary fibrosis in us medicare beneficiaries aged 65 years and older: Incidence, prevalence, and survival, 2001-11. The Lancet Respiratory medicine 2014;2(7):566-572.

6. Zank DC, Bueno M, Mora AL, Rojas M. Idiopathic pulmonary fibrosis: Aging, mitochondrial dysfunction, and cellular bioenergetics. Frontiers in medicine 2018;5:10.

7. Pardo A, Selman M. Lung fibroblasts, aging, and idiopathic pulmonary fibrosis. Annals of the American Thoracic Society 2016;13 Suppl 5:S417-S421.

8. Balch WE, Sznajder JI, Budinger S, Finley D, Laposky AD, Cuervo AM, Benjamin IJ, Barreiro E, Morimoto RI, Postow L, et al. Malfolded protein structure and proteostasis in lung diseases. Am J Respir Crit Care Med 2014;189(1):96-103.

9. Balch WE, Morimoto RI, Dillin A, Kelly JW. Adapting proteostasis for disease intervention. Science (New York, NY) 2008;319(5865):916-919.

10. Kropski JA, Blackwell TS. Progress in understanding and treating idiopathic pulmonary fibrosis. Annu Rev Med 2019;70:211-224.

11. Quiros PM, Prado MA, Zamboni N, D'Amico D, Williams RW, Finley D, Gygi SP, Auwerx J. Multiomics analysis identifies atf4 as a key regulator of the mitochondrial stress response in mammals. The Journal of cell biology 2017;216(7):2027-2045.

12. Pakos-Zebrucka K, Koryga I, Mnich K, Ljujic M, Samali A, Gorman AM. The integrated stress response. EMBO reports 2016;17(10):1374-1395.

13. Wootton SC, Kim DS, Kondoh Y, Chen E, Lee JS, Song JW, Huh JW, Taniguchi H, Chiu C, Boushey $\mathrm{H}$, et al. Viral infection in acute exacerbation of idiopathic pulmonary fibrosis. Am J Respir Crit Care Med 2011;183(12):1698-1702.

14. Moore BB, Paine R, 3rd, Christensen PJ, Moore TA, Sitterding S, Ngan R, Wilke CA, Kuziel WA, Toews GB. Protection from pulmonary fibrosis in the absence of ccr2 signaling. Journal of immunology 2001;167(8):4368-4377.

15. Gibbons MA, MacKinnon AC, Ramachandran P, Dhaliwal K, Duffin R, Phythian-Adams AT, van Rooijen N, Haslett C, Howie SE, Simpson AJ, et al. Ly6chi monocytes direct alternatively activated profibrotic macrophage regulation of lung fibrosis. American journal of respiratory and critical care medicine 2011;184(5):569-581.

16. Misharin AV, Morales-Nebreda L, Reyfman PA, Cuda CM, Walter JM, McQuattie-Pimentel AC, Chen CI, Anekalla KR, Joshi N, Williams KJN, et al. Monocyte-derived alveolar macrophages drive lung fibrosis and persist in the lung over the life span. The Journal of experimental medicine 2017;214(8):2387-2404.

17. McCubbrey AL, Barthel L, Mohning MP, Redente EF, Mould KJ, Thomas SM, Leach SM, Danhorn T, Gibbings SL, Jakubzick CV, et al. Deletion of c-flip from cd11b(hi) macrophages prevents development of bleomycin-induced lung fibrosis. American journal of respiratory cell and molecular biology 2018;58(1):66-78. 18. Joshi N, Watanabe S, Verma R, Jablonski RP, Chen CI, Cheresh P, Markov NS, Reyfman PA, McQuattie-Pimentel AC, Sichizya L, et al. A spatially restricted fibrotic niche in pulmonary fibrosis is sustained by $\mathrm{m}$-csf/m-csfr signalling in monocyte-derived alveolar macrophages. The European respiratory journal 2020;55(1).

19. Tighe RM, Liang J, Liu N, Jung Y, Jiang D, Gunn MD, Noble PW. Recruited exudative macrophages selectively produce cxcl10 after noninfectious lung injury. Am J Respir Cell Mol Biol 2011;45(4):781-788. 
20. Zhong Q, Zhou B, Ann DK, Minoo P, Liu Y, Banfalvi A, Krishnaveni MS, Dubourd M, Demaio L, Willis BC, et al. Role of endoplasmic reticulum stress in epithelial-mesenchymal transition of alveolar epithelial cells: Effects of misfolded surfactant protein. Am J Respir Cell Mol Biol 2011;45(3):498-509.

21. Nureki SI, Tomer Y, Venosa A, Katzen J, Russo SJ, Jamil S, Barrett M, Nguyen V, Kopp M, Mulugeta $\mathrm{S}$, et al. Expression of mutant sftpc in murine alveolar epithelia drives spontaneous lung fibrosis. The Journal of clinical investigation 2018;128(9):4008-4024.

22. Young LR, Borchers MT, Allen HL, Gibbons RS, McCormack FX. Lung-restricted macrophage activation in the pearl mouse model of hermansky-pudlak syndrome. J Immunol 2006;176(7):4361-4368.

23. Young LR, Gulleman PM, Bridges JP, Weaver TE, Deutsch GH, Blackwell TS, McCormack FX. The alveolar epithelium determines susceptibility to lung fibrosis in hermansky-pudlak syndrome. Am J Respir Crit Care Med 2012;186(10):1014-1024.

24. Venosa A, Katzen J, Tomer Y, Kopp M, Jamil S, Russo SJ, Mulugeta S, Beers MF. Epithelial expression of an interstitial lung disease-associated mutation in surfactant protein-c modulates recruitment and activation of key myeloid cell populations in mice. J Immunol 2019;202(9):2760-2771.

25. Seibold MA, Wise AL, Speer MC, Steele MP, Brown KK, Loyd JE, Fingerlin TE, Zhang W, Gudmundsson $\mathrm{G}$, Groshong SD, et al. A common muc5b promoter polymorphism and pulmonary fibrosis. $N$ Engl J Med 2011;364(16):1503-1512.

26. Adler M, Mayo A, Zhou X, Franklin R, Meizlish M, Medzhitov R, Kallenberger S, Alon U. Principles of cell circuits for tissue repair and fibrosis. bioRxiv 2019:710012.

27. Zhou X, Franklin RA, Adler M, Jacox JB, Bailis W, Shyer JA, Flavell RA, Mayo A, Alon U, Medzhitov R. Circuit design features of a stable two-cell system. Cell 2018;172(4):744-757 e717.

28. Chou A, Krukowski K, Jopson T, Zhu PJ, Costa-Mattioli M, Walter P, Rosi S. Inhibition of the integrated stress response reverses cognitive deficits after traumatic brain injury. Proceedings of the National Academy of Sciences of the United States of America 2017;114(31):E6420-E6426.

29. Sidrauski C, Acosta-Alvear D, Khoutorsky A, Vedantham P, Hearn BR, Li H, Gamache K, Gallagher $\mathrm{CM}$, Ang KK, Wilson C, et al. Pharmacological brake-release of mrna translation enhances cognitive memory. Elife 2013;2:e0498.

30. Adamson IY, Bowden DH. Response of mouse lung to crocidolite asbestos. 2. Pulmonary fibrosis after long fibres. $J$ Pathol 1987;152(2):109-117.

31. Misharin AV, Morales-Nebreda L, Mutlu GM, Budinger GR, Perlman H. Flow cytometric analysis of macrophages and dendritic cell subsets in the mouse lung. American journal of respiratory cell and molecular biology 2013;49(4):503-510.

32. Puchta A, Naidoo A, Verschoor CP, Loukov D, Thevaranjan N, Mandur TS, Nguyen PS, Jordana M, Loeb M, Xing Z, et al. Tnf drives monocyte dysfunction with age and results in impaired anti-pneumococcal immunity. PLoS Pathog 2016;12(1):e1005368.

33. Burman A, Tanjore H, Blackwell TS. Endoplasmic reticulum stress in pulmonary fibrosis. Matrix Biol 2018;68-69:355-365.

34. Burman A, Kropski JA, Calvi CL, Serezani AP, Pascoalino BD, Han W, Sherrill T, Gleaves L, Lawson WE, Young LR, et al. Localized hypoxia links er stress to lung fibrosis through induction of c/ebp homologous protein. JCI insight 2018;3(16):e99543.

35. Delbrel E, Soumare A, Naguez A, Label R, Bernard O, Bruhat A, Fafournoux P, Tremblais G, Marchant D, Gille T, et al. Hif-1alpha triggers er stress and chop-mediated apoptosis in alveolar epithelial cells, a key event in pulmonary fibrosis. Sci Rep 2018;8(1):17939.

36. Hsu HS, Liu CC, Lin JH, Hsu TW, Hsu JW, Su K, Hung SC. Involvement of er stress, pi3k/akt activation, and lung fibroblast proliferation in bleomycin-induced pulmonary fibrosis. Sci Rep 2017;7(1):14272. 37. Chen X, Nadiarynkh O, Plotnikov S, Campagnola PJ. Second harmonic generation microscopy for quantitative analysis of collagen fibrillar structure. Nat Protoc 2012;7(4):654-669.

38. Yona S, Kim K-W, Wolf Y, Mildner A, Varol D, Breker M, Strauss-Ayali D, Viukov S, Guilliams M, Misharin A, et al. Fate mapping reveals origins and dynamics of monocytes and tissue macrophages under homeostasis. Immunity 2013;38(1):79-91. 
39. McQuattie-Pimentel AC, Ren Z, Joshi N, Watanabe S, Stoeger T, Chi M, Lu Z, Sichizya L, Piseaux R, Chen C-I, et al. The aging microenvironment shapes alveolar macrophage identity in aging. bioRxiv 2019:717033.

40. Krukowski K, Nolan A, Frias ES, Grue K, Becker M, Ureta G, Delgado L, Bernales S, Sohal VS, Walter $\mathrm{P}$, et al. Integrated stress response inhibitor reverses sex-dependent behavioral and cell-specific deficits after mild repetitive head trauma. J Neurotrauma 2020.

41. Budinger GR, Mutlu GM, Eisenbart J, Fuller AC, Bellmeyer AA, Baker CM, Wilson M, Ridge K, Barrett TA, Lee VY, et al. Proapoptotic bid is required for pulmonary fibrosis. Proceedings of the National Academy of Sciences of the United States of America 2006;103(12):4604-4609.

42. Kang HR, Cho SJ, Lee CG, Homer RJ, Elias JA. Transforming growth factor (tgf)-beta1 stimulates pulmonary fibrosis and inflammation via a bax-dependent, bid-activated pathway that involves matrix metalloproteinase-12. The Journal of biological chemistry 2007;282(10):7723-7732.

43. Phan SH. The myofibroblast in pulmonary fibrosis. Chest 2002;122(6 Suppl):286S-289S.

44. Adler M, Mayo A, Zhou X, Franklin RA, Meizlish ML, Medzhitov R, Kallenberger SM, Alon U. Principles of cell circuits for tissue repair and fibrosis. iScience 2020;23(2):100841-100841.

45. Rosati A, Graziano V, De Laurenzi V, Pascale M, Turco MC. Bag3: A multifaceted protein that regulates major cell pathways. Cell death \& disease 2011;2(4):e141.

46. Hinnebusch AG, Lorsch JR. The mechanism of eukaryotic translation initiation: New insights and challenges. Cold Spring Harb Perspect Biol 2012;4(10):a011544.

47. Ameri K, Harris AL. Activating transcription factor 4. Int J Biochem Cell Biol 2008;40(1):14-21.

48. Mollereau B, Manie S, Napoletano F. Getting the better of er stress. J Cell Commun Signal 2014;8(4):311-321.

49. Nishitoh H. Chop is a multifunctional transcription factor in the er stress response. J Biochem 2012;151(3):217-219.

50. Rabouw HH, Langereis MA, Anand AA, Visser LJ, de Groot RJ, Walter P, van Kuppeveld FJM. Small molecule isrib suppresses the integrated stress response within a defined window of activation. Proceedings of the National Academy of Sciences of the United States of America 2019;116(6):2097-2102.

51. Sekine Y, Zyryanova A, Crespillo-Casado A, Fischer PM, Harding HP, Ron D. Stress responses. Mutations in a translation initiation factor identify the target of a memory-enhancing compound. Science 2015;348(6238):1027-1030.

52. Halliday M, Radford H, Sekine Y, Moreno J, Verity N, le Quesne J, Ortori CA, Barrett DA, Fromont C, Fischer PM, et al. Partial restoration of protein synthesis rates by the small molecule isrib prevents neurodegeneration without pancreatic toxicity. Cell death \& disease 2015;6:e1672.

53. Pandey VK, Mathur A, Khan MF, Kakkar P. Activation of perk-eif2 $\alpha$-atf4 pathway contributes to diabetic hepatotoxicity: Attenuation of er stress by morin. Cellular signalling 2019;59:41-52.

54. Li J, Akil O, Rouse SL, McLaughlin CW, Matthews IR, Lustig LR, Chan DK, Sherr EH. Deletion of tmtc 4 activates the unfolded protein response and causes postnatal hearing loss. J Clin Invest 2018;128(11):5150-5162.

55. Barragan-Iglesias P, Kuhn J, Vidal-Cantu GC, Salinas-Abarca AB, Granados-Soto V, Dussor GO, Campbell ZT, Price TJ. Activation of the integrated stress response in nociceptors drives methylglyoxalinduced pain. Pain 2019;160(1):160-171.

56. Wang C, Tan Z, Niu B, Tsang KY, Tai A, Chan WCW, Lo RLK, Leung KKH, Dung NWF, Itoh N, et al. Inhibiting the integrated stress response pathway prevents aberrant chondrocyte differentiation thereby alleviating chondrodysplasia. Elife 2018;7.

57. Nguyen HG, Conn CS, Kye Y, Xue L, Forester CM, Cowan JE, Hsieh AC, Cunningham JT, Truillet C, Tameire F, et al. Development of a stress response therapy targeting aggressive prostate cancer. Science translational medicine 2018;10(439):eaar2036.

58. Wong YL, LeBon L, Edalji R, Lim HB, Sun C, Sidrauski C. The small molecule isrib rescues the stability and activity of vanishing white matter disease eif2b mutant complexes. Elife 2018;7. 
59. Kabir ZD, Che A, Fischer DK, Rice RC, Rizzo BK, Byrne M, Glass MJ, De Marco Garcia NV, Rajadhyaksha AM. Rescue of impaired sociability and anxiety-like behavior in adult cacnalc-deficient mice by pharmacologically targeting eif2alpha. Molecular psychiatry 2017;22(8):1096-1109.

60. Briggs DI, Defensor E, Memar Ardestani P, Yi B, Halpain M, Seabrook G, Shamloo M. Role of endoplasmic reticulum stress in learning and memory impairment and alzheimer's disease-like neuropathology in the ps19 and app(swe) mouse models of tauopathy and amyloidosis. eNeuro 2017;4(4).

61. Johnson EC, Kang J. A small molecule targeting protein translation does not rescue spatial learning and memory deficits in the happ-j20 mouse model of alzheimer's disease. PeerJ 2016;4:e2565.

62. Kropski JA, Lawson WE, Young LR, Blackwell TS. Genetic studies provide clues on the pathogenesis of idiopathic pulmonary fibrosis. Disease models \& mechanisms 2013;6(1):9-17.

63. Young LR, Pasula R, Gulleman PM, Deutsch GH, McCormack FX. Susceptibility of hermansky-pudlak mice to bleomycin-induced type ii cell apoptosis and fibrosis. Am J Respir Cell Mol Biol 2007;37(1):67-74.

64. Povedano JM, Martinez P, Flores JM, Mulero F, Blasco MA. Mice with pulmonary fibrosis driven by telomere dysfunction. Cell reports 2015;12(2):286-299.

65. Naikawadi RP, Disayabutr S, Mallavia B, Donne ML, Green G, La JL, Rock JR, Looney MR, Wolters PJ. Telomere dysfunction in alveolar epithelial cells causes lung remodeling and fibrosis. JCI Insight 2016;1(14):e86704.

66. Ayaub EA, Kolb PS, Mohammed-Ali Z, Tat V, Murphy J, Bellaye PS, Shimbori C, Boivin FJ, Lai R, Lynn EG, et al. Grp78 and chop modulate macrophage apoptosis and the development of bleomycin-induced pulmonary fibrosis. $J$ Pathol 2016;239(4):411-425.

67. Yona S, Kim KW, Wolf Y, Mildner A, Varol D, Breker M, Strauss-Ayali D, Viukov S, Guilliams M, Misharin A, et al. Fate mapping reveals origins and dynamics of monocytes and tissue macrophages under homeostasis. Immunity 2013;38(1):79-91.

68. Madisen L, Zwingman TA, Sunkin SM, Oh SW, Zariwala HA, Gu H, Ng LL, Palmiter RD, Hawrylycz MJ, Jones AR, et al. A robust and high-throughput cre reporting and characterization system for the whole mouse brain. Nat Neurosci 2010;13(1):133-140.

69. Hubner RH, Gitter W, El Mokhtari NE, Mathiak M, Both M, Bolte H, Freitag-Wolf S, Bewig B. Standardized quantification of pulmonary fibrosis in histological samples. Biotechniques 2008;44(4):507-511, 514-507.

70. Kamp DW, Israbian VA, Yeldandi AV, Panos RJ, Graceffa P, Weitzman SA. Phytic acid, an iron chelator, attenuates pulmonary inflammation and fibrosis in rats after intratracheal instillation of asbestos. Toxicol Pathol 1995;23(6):689-695. 


\section{Acknowledgments:}

This work used services from Northwestern University Flow Cytometry Facility, Center For Advanced Microscopy, and Pathology Core Facility, which are supported by NCI Cancer Center Support Grant P30 CA060553 awarded to the Robert H. Lurie Comprehensive Cancer Center. Multiphoton microscopy was performed on a Nikon A1R multiphoton microscope, acquired through the support of NIH 1S10OD010398-01. This research was supported in part through the computational resources and staff contributions provided by the Genomics Computing Cluster (Genomic Nodes on Quest) which is jointly supported by the Feinberg School of Medicine, the Center for Genetic Medicine, and Feinberg's Department of Biochemistry and Molecular Genetics, the Office of the Provost, the Office for Research, and Northwestern Information Technology.

\section{Funding:}

Satoshi Watanabe is supported by MSD Life Science Foundation, Public Interest Incorporated Foundation, Japan, and David W. Cugell and Christina Enroth-Cugell Fellowship Program at Northwestern University. Paul A. Reyfman is supported by Northwestern University’s Lung Sciences Training Program 5T32HL07613913 and 1F32HL136111-01A1.

Rogan Grant is supported by T32AG020506-18.

GR Scott Budinger is supported by NIH grants ES013995, HL071643, AG049665, The Veterans Administration Grant BX000201.

Alexander V Misharin is supported by NIH grants HL135124, AG049665 and AI135964.

The authors declare no competing financial interests.

\section{Author contributions:}

Satoshi Watanabe: designed the study, performed experiments, analyzed results, wrote manuscript. Nikolay S. Markov: performed bioinformatics analysis, wrote manuscript. Ziyan Lu, Raul Piseaux Aillon, Saul Soberanes, Constance E. Runyan, Ziyou Ren, Rogan A. Grant, Mariana Maciel, Hiam Abdala-Valencia, Yuliya Politanska, Kiwon Nam, Lango Sichizya, Hermon G. Kihshen, Nikita Joshi, Alexandra C. McQuattie-Pimentel, Paul A. Reyfman: performed experiments, analyzed results. Richard I. Morimoto: designed and supervised the study, performed analysis, wrote manuscript. GR Scott Budinger and Alexander V. Misharin: designed and supervised the study, performed analysis, wrote manuscript, provided funding for the project. All authors discussed the results and commented on the manuscript. 